\title{
Scare Behavior Diffusion Model of Health Food Safety Based on Complex Network
}

\author{
Jun Luo $\mathbb{D}^{1,2}$ Jiepeng Wang, ${ }^{3}$ Yongle Zhao, ${ }^{1}$ and Tingqiang Chen $\mathbb{D}^{3}$ \\ ${ }^{1}$ Business School, Hohai University, Nanjing, China \\ ${ }^{2}$ School of Health Economics and Management, Nanjing University of Chinese Medicine, Nanjing, China \\ ${ }^{3}$ School of Economics and Management, Nanjing Tech University, Nanjing, China \\ Correspondence should be addressed to Tingqiang Chen; tingqiang88888888@163.com
}

Received 26 July 2018; Revised 30 September 2018; Accepted 15 October 2018; Published 1 November 2018

Guest Editor: Piotr Brodka Copyright (C) 2018 Jun Luo et al. This is an open access article distributed under the Creative Commons Attribution License, which
permits unrestricted use, distribution, and reproduction in any medium, provided the original work is properly cited.

\begin{abstract}
This study constructs a heterogeneous model of health food safety scare behavior diffusion through a complex network model by considering health food safety information transparency and health food consumers' ability to process information. This study first analyzes the effects of network structure and heterogeneity of health food consumers on the health food safety scare behavior diffusion using network stochastic dominance theory. Subsequently, a computer mathematical simulation is performed to explore the characteristics and laws of the evolution of health food safety scare behavior diffusion. The following three major conclusions can be drawn from the results. First, increases in the health food safety information transparency, the health food consumers' ability to process information, and the recovery rate of health food consumers can increase the threshold of the rate of health food safety scare behavior diffusion. The health food safety information transparency and the recovery rate of health food consumers show marginal incremental rising characteristics in relation to the rate of health food safety scare behavior diffusion, whereas the health food consumers' ability to process information shows a marginal diminishing rising characteristic in relation to the rate of health food safety scare behavior diffusion. Second, increases in the health food safety information transparency, the health food consumers' ability to process information, and the recovery rate of health food consumers can decrease the scale of the health food safety scare behavior diffusion. The health food safety information transparency shows a marginal diminishing decreasing characteristic in relation to the scale of the health food safety scare behavior diffusion, whereas the health food consumers' ability to process information and the recovery rate of the health food consumers show marginal incremental decreasing characteristics in relation to the scale of the health food safety scare behavior diffusion. Finally, the network structure of health food consumers significantly affects the health food safety scare behavior diffusion. A high heterogeneity of the health food consumer network indicates a high threshold of the rate of health food safety scare behavior diffusion and low diffusion scale.
\end{abstract}

\section{Introduction}

The concept of food safety is continuously developing. Understanding food safety is a dynamic development process [15]. Food safety includes not only food security but also food quality and health safety [3-5]. In recent years, numerous media reports on food industry emergencies have been persistent, thereby transforming a sudden food safety problem into a serious public policy and social problem. For example, incidents related to salt supply safety broke out from March 16, 2011, to March 18, 2011, under the influence of scare behavior diffusion in China after the 2011 earthquake in
Japan. The food safety scare behavior caused by food safety accidents can spread through certain media to consumers in their healthy state as a product of the development and evolution of emergencies [3-6], thereby provoking panic behavior with a significant herd effect. Many food safety accidents are safe in themselves, but the loss induced by these accidents is much greater than the direct loss from the accidents themselves $[7,8]$. Therefore, food safety accidents are detrimental to the healthy development of social stability and food industry $[9,10]$.

Currently, the party and the government in China introduced the plan "Healthy China 2030." The development of 
new business forms of the health industry, as an important part of "Healthy China 2030," must be considered. The health food industry, as an emerging health industry, has numerous apparent shortcomings, including the imperfection of the industrial system, low level of the industrial system, low coordination effect, and lack of industry standards and norms [11]; consequently, the health food industry has received extensive attention from the society. Specifically, chaos may result from health food safety accidents, such as those in which health food passes off as medicine, health food contains illegal drug components, and functional efficacy of health food is inconsistent with reality. The health food safety scare behavior caused by such accidents has affected the implementation of China's strategy of building a healthy China. Furthermore, existing empirical studies show that improving the transparency of food safety supervision information can reduce the adverse effect of food safety accidents [3-5, 1217]. Mol [16] investigated to what extent and how China's transparency institutions and practices regarding food production and products play a role in governing food quality and safety. Chen et al. [3-5] found that the transparency of food safety supervision in China is basically qualified but remains at a poor level; this transparency may be improved by establishing an index system for food safety supervision information transparency and evaluating different regulatory bodies in China. Easing the rumor-driven "herd behavior" is not conducive to promoting the healthy development of food industry. The role of food information transparency in alleviating the food safety problem has gradually attracted the attention of numerous scholars given the reinforcement of food safety management $[3-5,16,18]$. However, health food is in the primary stage of development in China. Moreover, research on information transparency in the health food industry is limited; let alone research on health food safety scare behavior with consideration of health food information transparency. Therefore, in accordance with the suggestion of Chen et al. [3-5], two key factors, namely, health food information transparency and health food consumers' ability to process information, are considered in the current study by exploring the internal mechanism and evolution law of the health food safety scare behavior diffusion among health food consumers. The results of the present study can change the current situation of health food safety in China to a certain extent and promote the formation of social cogovernance of health food safety.

Complex network theory and methods have been developed in several studies [19-24] and have been applied to various fields. A network is a collection of nodes and edges. A complex network is composed of many nodes and edges of connecting nodes. Its complexity is mainly manifested in the number and properties of the nodes and edges. It is usually a high generalization or abstraction of complex systems and phenomena. Compared with a general network, a complex network has six unique characteristics that reflect its complexity. First, a complex network has a complicated structure. The number of its nodes is large, and the network structure presents many different characteristics. Second, a complex network presents network evolution, as reflected in the emergence and disappearance of nodes or connections.
Third, a complex network has a connection diversity. The connection weights between nodes vary, and the connections may be directional. Fourth, a complex network has a dynamic complexity. A node set may belong to a nonlinear dynamic system. Fifth, a complex network features a node diversity. Nodes in complex networks can represent anything. Finally, a complex network presents multiple-complexity integration. The complexities that result from the five aforementioned characteristics influence one another, and their interplay leads to unpredictable results. In addition to these six complex characteristics, a complex network also has a dynamic complexity in time and space, and its network behavior is also complex. Many real-world complex systems, such as transportation network, the Internet, investment network, and disease contagion network, can be in the form of a complex network by abstracting, description, characterization, and analysis. The capability of complex networks to represent real-world phenomena and their dynamic evolution behaviors has prompted science researchers in various fields to describe, analyze, and model complex networks and establish algorithms thereof in theoretical and empirical studies. By the end of the 20th century, the WS network proposed by Watts and Strogatz [19] and the BA network proposed by Barabási and Albert [20] epitomize the new era of complex network research. At present, complex network theory, which is the general methodology for analyzing all types of complex systems and their complex phenomena in the real world, has infiltrated studies in many fields, including statistical physics, biological sciences, and humanistic social sciences [25-27]. Insights into and methods based on complex networks have become a research interest in the scientific study community.

The complex network constructed in this study is the health food consumer network. Currently, health food consumers in China are mainly the elderly, and the health food consumer network becomes increasingly complex while the aging population in China expands. On the one hand, health food consumers exhibit different cognitive performances in terms of health food [3-5, 28, 29]. Several health food consumers are protected from health food safety scare behavior considering their abundant knowledge of health food; these consumers can inhibit the spread of this behavior. By contrast, certain health food consumers are vulnerable to the influence of connected health food consumers, thereby showing remarkable herd behavior [30]. On the other hand, various health food consumers possess different psychological qualities or psychological cognitions. In the face of health food safety scare behavior, consumers with high psychological quality can be minimally affected or can even avoid being affected by this scare behavior. In consideration of this scenario, this study investigates health food consumers' ability to process information. Heterogeneity in such a complex network cannot be ignored. Therefore, this study uses stochastic dominant theory to analyze the health food safety scare behavior diffusion under different network structures.

The concept of stochastic dominance was first explicitly proposed by Quirk and Saposnik in 1962 [31], and they associated it with the traditional expected utility principle. Since then, stochastic dominance theory has been the basis of risk decision methods. This theory has been a widely 


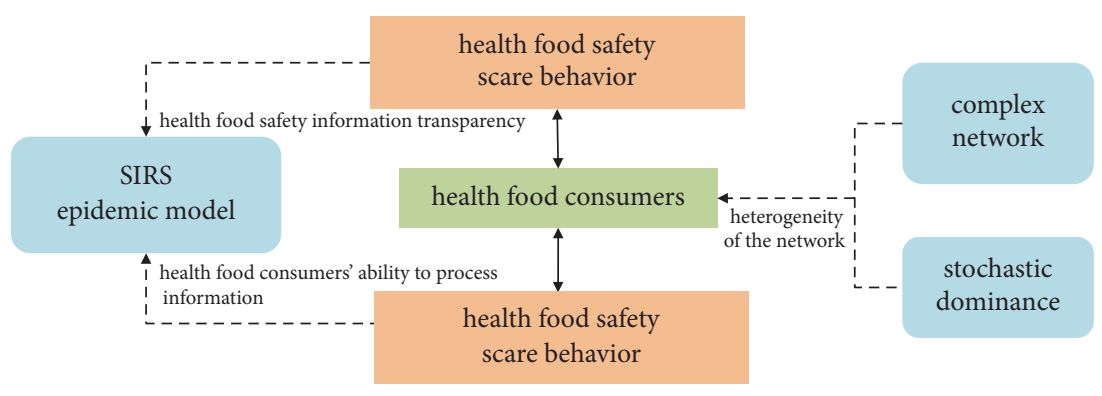

FIgURE 1: Technology roadmap of the article.

recognized decision analysis tool used by economic agents to make a decision of behavior in uncertainty cases. Stochastic dominance is a nonparametric decision analysis method in which various optional results and their corresponding objective probabilities are analyzed to filter the nondominant schemes and make risk decisions. Studies on stochastic dominance date back to Karamata [32]. However, the theory attracted the attention of the academic community only in the late 1960s and early 1970s when the studies of Hadar and Russel [33], Hanoch and Levy [34], and Rothchild and Stiglitz [35] were reported. Particularly in the 1970s, numerous studies on stochastic dominance theory and its applications emerged in the research literature. Several of these studies proposed decision-making methods, such as the third-order and expectation stochastic dominance. The most important representatives of these studies is that of Fishburn [36], who extended the concept of stochastic dominance to random variables. Jackson [37] adopted the stochastic dominance method to analyze a social network structure and investigated the influence of structural characteristics and network heterogeneity on social behavior. Bian et al. [38] used the stochastic dominance to explore the evolvement of investors' behavior in stock market. In the present study, we use the same method used by Jackson [37] to analyze the influence of structure and heterogeneity of the health food consumer network on the health food safety scare behavior diffusion.

Health food safety scare behavior diffusion is a typical proliferation problem, and its mechanism is similar to those of the spread of infectious diseases. The technology roadmap of this study is illustrated in Figure 1. We establish a network diffusion model on the basis of the SIRS model of health food safety scare behavior and analyze the influences of health food information transparency, health food consumers' ability to process information, and network structure on the mechanism of health food safety scare behavior diffusion. This study can provide a reference for controlling health food safety scare behavior diffusion and reducing its effect on the society.

The structure of this study is arranged as follows. Section 2 discusses the infectious disease principles and characteristics of health food safety scare behavior diffusion in two aspects: (1) adaptability of the epidemic model and (2) health food safety scare behavior diffusion mechanism. Section 3 presents the model constructed for health food safety scare behavior diffusion and the corresponding theoretical analysis. Section 4 presents a computer simulation analysis of the health food safety scare behavior diffusion. Section 5 summarizes the conclusion of this study.

\section{Infectious Disease Principles and Characteristics of Health Food Safety Scare Behavior Diffusion}

2.1. Adaptability Analysis of the Epidemic Model. The epidemic model, as a classical model of virus transmission, has been extensively used in studying social behavior diffusion $[3-5,39-41]$. The source of an infectious disease is a virus carrier or a pathogen, which spreads its own virus by a contact through a certain medium [42]. Health food safety scare behavior diffusion, what we define is the interaction and transmission of the behavior of health food consumers. It denotes that the scare behavior of health food consumers will spread through all kinds of media to health food consumers who are uninfected. The health food safety scare behavior affects stakeholders like a virus, and many mechanisms are similar between the processes of diffusion and virus propagation. The principal representations are as follows:

(1) Pathogen-diffusion source. The spread of health food safety scare mainly stems from the concerns of health food consumers over health food safety [43]. With the influence of factors, such as health food information transparency, health food consumers' ability to process information, and network structure, health food consumers who produce health food safety scare behavior are "pathogens" or diffusion sources that have the potential to spread. A diffusion source, which is a prerequisite for health food safety scare behavior diffusion, will spread health food safety scare behavior to health food consumers through the diffusion media, thereby presenting a significant herding effect.

(2) Contagion medium-diffusion medium. A diffusion medium is the carrier of the diffusion source, such as the Internet, mobile phone, TVs, and other mass media, and face-to-face communication between health food consumers. The health food safety information transmitted through the diffusion media is related to the health and safety of health food consumers. The transparency of health food safety information affects the confidence of health food consumers in health food safety $[3-5,16,17]$.

(3) Infectiousness. Health food consumers involved in the health food safety scare deliver their psychological cognition, behavior deviation, and other information to health food 


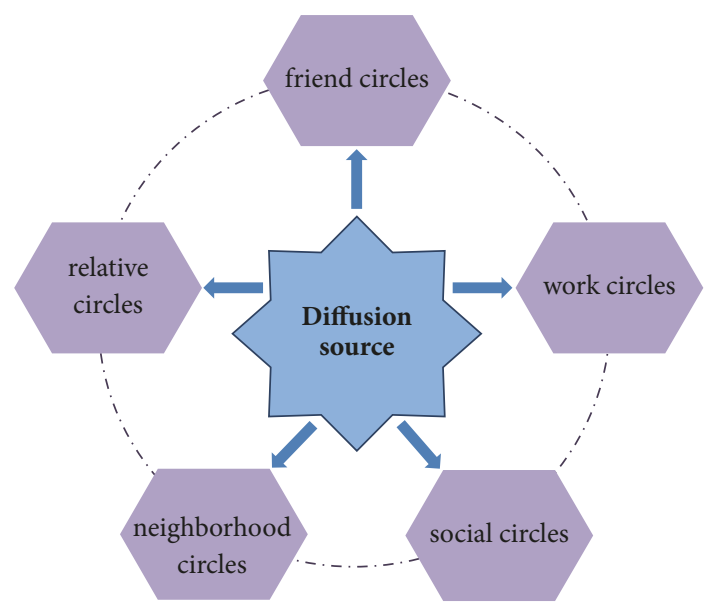

Figure 2: Diffusion media and diffusion path of health food safety scare behavior.

consumers who are in a healthy state through the diffusion media given the influence of health food safety information transparency, thereby inducing the latter to divert their attention and psychological cognition to the health food safety accident and consequently causing scare behavior. This mechanism shows that the health food safety scare behavior has certain infectiousness. In Figure 2, health food consumers that exhibit health food safety scare behavior will pass information, such as their own psychological state and behavioral biases to the external environment, thus affecting the health food consumers in a normal state and causing them to panic.

(4) Immunity. In the epidemic model, individuals are immune to pathogens. Health food consumers show different immunity levels to health food safety scare behavior given their different degrees of psychological quality and health food safety knowledge as influenced by the health food safety regulatory information transparency. If the health food information transparency is considerable, the psychological quality of health food consumers is high, and the health food safety knowledge of health food consumers abounds, then the health food safety scare behavior diffusion will be suppressed. Otherwise, the health food safety scare behavior diffusion will be accelerated.

Therefore, the scare behavior diffusion process of health food industry emergencies is characterized by the infectious disease propagation process, which can be used to analyze and simulate the process of the scare behavior diffusion for health food. Therefore, constructing an SIRS epidemic model of the health food safety scare behavior diffusion is reasonable.

\subsection{Health Food Safety Scare Behavior Diffusion Mechanism.} In a complex network of $N$ health food consumers, the nodes represent health food consumers, and the edges represent the diffusion media between two health food consumers. Several health food consumers exhibit scare behavior, whereas other health food consumers have the potential of adopting the behavior. Accordingly, spreading the health food safety scare

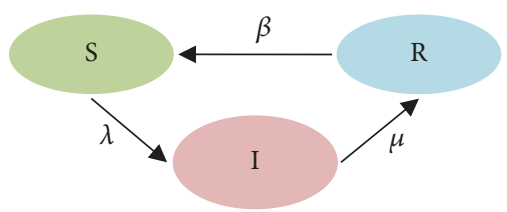

Figure 3: Health food safety scare behavior diffusion mechanism.

behavior is realized through such a complex network. Degree $k$ represents the relationship between health food consumers and also denotes the number of diffusion media and paths.

In the network of health food consumers, each health food consumer exhibits one of the following states:

(1) Normal state $S$. This state indicates that health food consumers are unaffected by the health food safety scare behavior but may contract it.

(2) Scare state $I$. This state indicates that health food consumers have contracted the health food safety scare behavior by diffusion and are contagious.

(3) Temporary immunity state $R$. This state indicates that health food consumers have recovered to normal states and have the ability to be immune from health food safety scare behavior temporarily; however, these consumers will be in a vulnerable normal state again after a time and continue to be affected by the health food safety scare behavior.

At the beginning, no health food safety incident has occurred in the health food consumer network. A health food consumer in the network is either in the normal state $S$, scare state $I$, or temporary immunity state $R$ when a health food emergency occurs. At moment $t$, the proportion of health food consumers who are in the normal state in the network is called the normal state health food consumer density, which is denoted by $s(t)$. The proportion of the health food consumers who are in the scare state in the network is called the scare state health food consumer density, which is denoted by $i(t)$. The proportion of health food consumers who are in the temporary immunity state in the network is called the temporary immunity state health food consumer density, which is denoted by $r(t)$. Therefore, $s(t)+i(t)+$ $r(t)=1$. The network of health food consumers tends to be balanced when $t \longrightarrow \infty$. $I$ is the origin of diffusion that spreads to $S$ at probability $\lambda$ (diffusion rate) and recovers to be $R$ at probability $\mu$ (recovery rate) simultaneously. $R$ loses immunity at probability $\beta$ (immune failure rate) to be $S$ (Figure 3).

The health food safety scare behavior diffusion between health food consumers is uneven. This unevenness is related to the behavior diffusion rate, the health food safety information transparency, and the health food consumers' ability to process information.

(1) Health food safety information transparency. According to Chen et al. [3-5], the transparency of food safety information is an important factor that affects food safety risk. Thus, for health food, a high transparency of health food safety information denotes an improved delivery or disclosure of the information on health food by the government, enterprises, and media. The spread of health food 
TABLE 1: Sensitivity analysis of the health food safety information transparency $\theta$ and the degree $k$ of health food consumers' ability to process information $\eta_{k}$ to the health food safety scare behavior diffusion rate $\lambda_{k}$.

\begin{tabular}{|c|c|c|c|c|c|c|c|c|c|c|c|}
\hline \multirow{2}{*}{$\theta$} & \multicolumn{9}{|c|}{$\eta_{k}$} & \multirow{2}{*}{ Expectation } & \multirow{2}{*}{ Variance } \\
\hline & 0.1 & 0.2 & 0.3 & 0.4 & 0.5 & 0.6 & 0.7 & 0.8 & 0.9 & & \\
\hline 0.1 & $5.60 \mathrm{E}-01$ & 3.39E-01 & $2.10 \mathrm{E}-01$ & $1.33 \mathrm{E}-01$ & 8.48E-02 & $5.46 \mathrm{E}-02$ & $3.54 \mathrm{E}-02$ & $2.31 \mathrm{E}-02$ & $1.52 \mathrm{E}-02$ & $1.62 \mathrm{E}-01$ & $3.34 \mathrm{E}-02$ \\
\hline 0.2 & 4.82E-01 & $2.81 \mathrm{E}-01$ & $1.72 \mathrm{E}-01$ & 1.09E-01 & 7.10E-02 & 4.69E-02 & $3.14 \mathrm{E}-02$ & $2.12 \mathrm{E}-02$ & $1.45 \mathrm{E}-02$ & 1.37E-01 & $2.42 \mathrm{E}-02$ \\
\hline 0.3 & $3.99 \mathrm{E}-01$ & $2.25 \mathrm{E}-01$ & $1.38 \mathrm{E}-01$ & $8.85 \mathrm{E}-02$ & $5.87 \mathrm{E}-02$ & 3.99E-02 & 2.77E-02 & $1.95 \mathrm{E}-02$ & $1.39 \mathrm{E}-02$ & $1.12 \mathrm{E}-01$ & $1.62 \mathrm{E}-02$ \\
\hline 0.4 & $3.15 \mathrm{E}-01$ & $1.73 \mathrm{E}-01$ & $1.07 \mathrm{E}-01$ & 7.01E-02 & $4.79 \mathrm{E}-02$ & 3.37E-02 & $2.43 \mathrm{E}-02$ & $1.78 \mathrm{E}-02$ & $1.33 \mathrm{E}-02$ & 8.91E-02 & $9.79 \mathrm{E}-03$ \\
\hline 0.5 & $2.33 \mathrm{E}-01$ & $1.28 \mathrm{E}-01$ & 8.03E-02 & $5.43 \mathrm{E}-02$ & $3.85 \mathrm{E}-02$ & $2.82 \mathrm{E}-02$ & $2.12 \mathrm{E}-02$ & $1.63 \mathrm{E}-02$ & $1.27 \mathrm{E}-02$ & $6.80 \mathrm{E}-02$ & $5.19 \mathrm{E}-03$ \\
\hline 0.6 & $1.60 \mathrm{E}-01$ & 8.90E-02 & 5.81E-02 & $4.11 \mathrm{E}-02$ & $3.05 \mathrm{E}-02$ & $2.34 \mathrm{E}-02$ & $1.84 \mathrm{E}-02$ & $1.48 \mathrm{E}-02$ & $1.21 \mathrm{E}-02$ & 4.97E-02 & $2.31 \mathrm{E}-03$ \\
\hline 0.7 & $9.95 \mathrm{E}-02$ & 5.83E-02 & $4.04 \mathrm{E}-02$ & $3.02 \mathrm{E}-02$ & $2.37 \mathrm{E}-02$ & $1.92 \mathrm{E}-02$ & $1.60 \mathrm{E}-02$ & $1.35 \mathrm{E}-02$ & $1.15 \mathrm{E}-02$ & $3.47 \mathrm{E}-02$ & $8.13 \mathrm{E}-04$ \\
\hline 0.8 & $5.47 \mathrm{E}-02$ & $3.55 \mathrm{E}-02$ & $2.68 \mathrm{E}-02$ & $2.16 \mathrm{E}-02$ & $1.82 \mathrm{E}-02$ & $1.56 \mathrm{E}-02$ & $1.37 \mathrm{E}-02$ & $1.22 \mathrm{E}-02$ & $1.10 \mathrm{E}-02$ & $2.33 \mathrm{E}-02$ & $2.00 \mathrm{E}-04$ \\
\hline 0.9 & $2.58 \mathrm{E}-02$ & $1.98 \mathrm{E}-02$ & $1.69 \mathrm{E}-02$ & $1.50 \mathrm{E}-02$ & $1.36 \mathrm{E}-02$ & $1.26 \mathrm{E}-02$ & $1.18 \mathrm{E}-02$ & $1.11 \mathrm{E}-02$ & $1.05 \mathrm{E}-02$ & $1.52 \mathrm{E}-02$ & $2.46 \mathrm{E}-05$ \\
\hline
\end{tabular}

safety scare behavior will be suppressed when health food consumers provide sufficient information. Otherwise, the spread of health food safety scare behavior will be promoted.

(2) Health food consumers' ability to process information. On the one hand, health food consumers exhibit different cognitive performances in terms of health food. Several health food consumers are unaffected by the scare behavior and inhibit the spread of this behavior given their knowledge of health food. However, several health food consumers are vulnerable to the influence of connected health food consumers, thereby demonstrating a remarkable herd behavior. On the other hand, different health food consumers possess various mental qualities and therefore show different psychological qualities and psychological cognitions. Health food consumers with high psychological quality are less or even unaffected by scare behavior. Therefore, a high level of health food consumers' ability to process information exerts a certain inhibitory effect on the health food safety scare behavior diffusion.

The given analysis shows that the diffusion rate in the proposed model of the health food safety scare behavior is as follows:

$$
\lambda_{k}\left(\lambda_{0}, \theta, \eta\right)=\lambda_{0}^{\theta^{1-\eta_{k}}},
$$

where $\lambda_{0}$ denotes the diffusion rate that satisfies the condition $0<\lambda_{0}<1$ and $\theta$ denotes the health food safety information transparency that satisfies the condition $0<\theta<1$. A high $\theta$ indicates a transparent health food safety information. $\eta_{k}$ denotes the degree $k$ of health food consumers' ability to process information. A high $\eta_{k}$ denotes that health food consumers can process information. To better analyze the diffusion rate mode, we make visualization of the model via Matlab2016b. Based on that, we also make the sensitivity analysis. The results are shown as in Figure 4 and Table 1.

Figure 4 depicts the effects of the health food safety information transparency $\theta$ and the degree $k$ of health food consumers' ability to process information $\eta_{k}$ on the health food safety scare behavior diffusion rate $\lambda_{k}$. The health food safety scare behavior diffusion rate $\lambda_{k}$ decreases nonlinearly with an increase in the health food safety information transparency $\theta$ or the degree $k$ of health food consumers' ability to

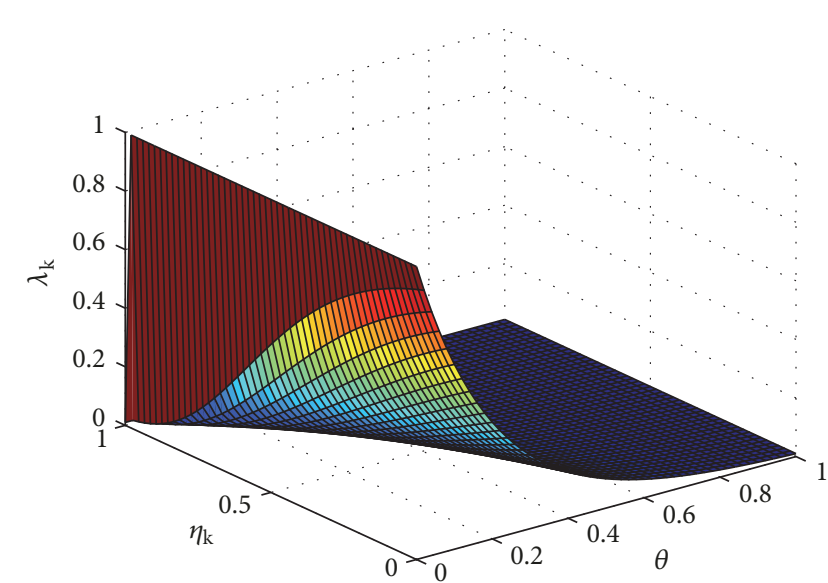

FIGURE 4: Effects of the health food safety information transparency $\theta$ and the degree $k$ of health food consumers' ability to process information $\eta_{k}$ on the health food safety scare behavior diffusion rate $\lambda_{k}$ when $\lambda_{0}=0.01$ and $k=1000$.

process information $\eta_{k}$. In the sensitivity analysis displayed in Table 1, the health food safety information transparency $\theta$ reflects a marginal diminishing decreasing characteristic in relation to the health food safety scare behavior diffusion rate $\lambda_{k}$, whereas the degree $k$ of health food consumers' ability to process information $\eta_{k}$ shows a marginal incremental decreasing characteristic in relation to the health food safety scare behavior diffusion rate $\lambda_{k}$. These results are consistent with the facts. Therefore, the established diffusion rate model on health food safety scare can be reasonably constructed.

\section{Diffusion Model of Health Food Safety Scare Behavior}

3.1. Model Construction. If the density of degree $k$ of health food consumers in the scare state is $i_{k}(t)$ at time $t$, then the density of its equilibrium state is $i_{k}(\infty) . s_{k}(\infty)$ and $r_{k}(\infty)$ have the same definition. According to mean-field theory (Moreno et al., 2003; Yang et al., 2006), the dynamics model 
of health food safety scare behavior diffusion in this network is as follows:

$$
\begin{aligned}
& s_{k}^{\prime}(t)=-k \lambda_{k} \Theta(t) s_{k}(t)+\beta r_{k}(t) \\
& i_{k}^{\prime}(t)=k \lambda_{k} s_{k}(t) \Theta(t)-\mu i_{k}(t) \\
& r_{k}^{\prime}(t)=\mu i_{k}(t)-\beta r_{k}(t),
\end{aligned}
$$

where $\Theta(t)$ represents the probability that a health food consumer in the normal state is directly linked to a health food consumer in the scare state. In equilibrium, the stable value is denoted by $\Theta(\infty)$. The first term on the right side of the first line of (2) is the health food safety scare behavior production item. This term is proportional to the degree $k$ of health food consumers, the health food safety scare behavior diffusion rate $\lambda_{k}$, and the density $s_{k}(t)$ of degree $k$ of health food consumers in the normal state at time $t$. The second term is the health food safety scare behavior annihilation term, which is proportional to the immune failure rate $\beta$ and the density $r_{k}(t)$ of degree $k$ of health food consumers in the temporary immunity state at time t. $\Theta(t)$ is defined as follows:

$$
\Theta(t)=\frac{\sum_{k} k P(k) i_{k}(t)}{\langle k\rangle} .
$$

Equation (3) represents the probability that a health food consumer in the normal state is directly linked to a health food consumer in the scare state at time $t ;\langle k\rangle=\sum_{k} k P(k)$ denotes the average degree of the network.

Setting $\left\{\begin{array}{l}s_{k}^{\prime}(t)=0 \\ i_{k}^{\prime}(t)=0 \\ r_{k}^{\prime}(t)=0\end{array} \quad\right.$ yields the following unsteady solution:

$$
i_{k}(\infty)=\frac{\lambda_{k} k \Theta(\infty)}{\lambda_{k} k \Theta(\infty)(1+\mu / \beta)+\mu} .
$$

The following equation can be obtained by substituting (4) into (3):

$$
\begin{aligned}
\Theta(\infty) & =\frac{\sum_{k} k P(k)}{\langle k\rangle} \frac{\lambda_{k} k \Theta(\infty)}{\lambda_{k} k \Theta(\infty)(1+\mu / \beta)+\mu} \\
& =G(\Theta(\infty)),
\end{aligned}
$$

where $\Theta(\infty)=0$ is a trivial solution in (5); that is, the health food safety scare behavior does not spread widely. If the health food safety scare behavior diffusion occurs, then (5) must have a nontrivial solution that satisfies $\left.(d G(\Theta(\infty)) / d \Theta(\infty))\right|_{\Theta(\infty)=0} \geq 1 . \lambda_{k}\left\langle k^{2}\right\rangle / \mu\langle k\rangle \geq 1$ can be obtained through simplification. Therefore, the critical condition of the health food safety scare behavior diffusion rate can be obtained as follows:

$$
\lambda_{0}^{\theta^{1-\eta_{k}}}=\frac{\mu\langle k\rangle}{\left\langle k^{2}\right\rangle} .
$$

Accordingly, we can obtain the threshold of the rate of health food safety scare behavior diffusion $\lambda_{0} *=(\mu\langle k\rangle /$ $\left.\left\langle k^{2}\right\rangle\right)^{\theta^{\eta_{k}-1}}$, where $\left\langle k^{2}\right\rangle=\sum_{k} k^{2} P(k)$. Finally, we can derive the entire health food consumer network diffusion as follows:

$$
i(\infty)=\sum_{k} P(k) i_{k}(\infty) .
$$

3.2. Model Analysis. In accordance with the established model in Section 3.1, this study analyzes the threshold of the rate of health food safety scare behavior diffusion; that is, $\lambda_{0} *=\left(\mu\langle k\rangle /\left\langle k^{2}\right\rangle\right)^{\theta^{\eta_{k}-1}}$, in accordance with the following relationships.

(1) Relationship to the Health Food Safety Information Transparency $\theta$

$$
\begin{aligned}
\frac{\partial \lambda_{0} *}{\partial \theta} & =\left(\eta_{k}-1\right) \cdot\left(\frac{\mu\langle k\rangle}{\left\langle k^{2}\right\rangle}\right)^{\theta^{\eta_{k}-1}} \cdot \ln \left(\frac{\mu\langle k\rangle}{\left\langle k^{2}\right\rangle}\right) \cdot \theta^{\eta_{k}-2} \\
& >0
\end{aligned}
$$

(2) Relationship to the Degree $k$ of Health Food Consumers' Ability to Process Information $\eta_{k}$

$$
\frac{\partial \lambda_{0} *}{\partial \eta_{k}}=\left(\frac{\mu\langle k\rangle}{\left\langle k^{2}\right\rangle}\right)^{\theta^{\eta_{k}-1}} \cdot \ln \left(\frac{\mu\langle k\rangle}{\left\langle k^{2}\right\rangle}\right) \cdot \theta^{\eta_{k}-1} \ln \theta>0
$$

(3) Relationship to the Recovery Rate $\mu$ of Health Food Consumers

$$
\begin{aligned}
\frac{\partial \lambda_{0} *}{\partial \mu} & =\left(\frac{\langle k\rangle}{\left\langle k^{2}\right\rangle}\right)^{\theta^{\eta_{k}-1}} \cdot \theta^{\eta_{k}-1} \cdot \mu^{\theta^{\eta_{k}-1}-1}>0 \\
\frac{\partial^{2} \lambda_{0} *}{\partial \mu^{2}} & =\left(\frac{\langle k\rangle}{\left\langle k^{2}\right\rangle}\right)^{\theta^{\eta_{k}-1}} \cdot \theta^{\eta_{k}-1} \cdot\left(\theta^{\eta_{k}-1}-1\right) \mu^{\theta^{\eta_{k}-1}-2} \\
& >0
\end{aligned}
$$

In summary, the following theorem is obtained.

Theorem 1. The threshold $\lambda_{0} *$ of the rate of health food safety scare behavior diffusion is the monotonically increasing function of the health food safety information transparency $\theta$, the degree $k$ of health food consumers' ability to process information $\eta_{k}$, and the monotonically increasing convex function of the recovery rate $\mu$ of health food consumers.

(4) Relationship to Different Network Structures. Let $P^{\prime}$ and $P$ represent the degree distribution of the two health food consumer networks $K^{\prime}$ and $K$, respectively.

$\left.G^{\prime}(\Theta(\infty))=\left\langle k^{2}\right\rangle \lambda_{k} \mu /\langle k\rangle\left(\lambda_{k} k \Theta(\infty)(1+\mu / \beta)+\mu\right)^{2}\right\rangle$ 0 and $G^{\prime \prime}(\Theta(\infty))=-\left\langle k^{2}\right\rangle \lambda_{k}{ }^{2} k(1+\mu / \beta) /\langle k\rangle\left(\lambda_{k} k \Theta(\infty)(1+\right.$ $\mu / \beta)+\mu)^{4}<0$. Thus, $G(\Theta(\infty))$ is the monotonically increasing concave function of $\Theta(\infty) . G(1)=\left(\sum_{k} k P(k) /\right.$ $\langle k\rangle)\left(\lambda_{k} k /\left(\lambda_{k} k(1+\mu / \beta)+\mu\right)\right)<\left(\sum_{k} k P(k) /\langle k\rangle\right)\left(\lambda_{k} k / \lambda_{k} k\right)=1$ and $G(0)=0$. Thus, $G(\Theta(\infty))$ has at least one fixed point in the interval $[0,1]$. Health food safety scare behavior spreads to the entire consumer network, and the network reaches the equilibrium state when $\lambda_{0}>\lambda_{0} *$. Simultaneously, $\left.G^{\prime}(\Theta(\infty))\right|_{\Theta=0}>1$. Therefore, $\Theta(\infty)=\left(\left\langle k^{2}\right\rangle /\right.$ $\langle k\rangle)\left(\lambda_{k} \Theta(\infty) /\left(\lambda_{k} k \Theta(\infty)(1+\mu / \beta)+\mu\right)\right)$ has the only equilibrium point in the interval $[0,1]$, and $\Theta(\infty)^{*}>0$.

The following theorems can be obtained in accordance with the criteria of stochastic dominance.

Theorem 2. If the average degree $\left\langle K^{\prime}\right\rangle$ of the health food consumer network $K^{\prime}$ is greater than the average degree $\langle K\rangle$ of 
the health food consumer network $K$, then (1) the equilibrium value $\Theta(\infty)^{* \prime}$ of the health food safety scare behavior diffusion in network $K^{\prime}$ is greater than the equilibrium value $\Theta(\infty){ }^{*}$ of the health food safety scare behavior diffusion in network $K$ when $\lambda_{0}>\lambda_{0} *$; moreover, (2) the scale $i(\infty)^{* \prime}$ of the health food safety scare behavior diffusion in network $K^{\prime}$ is greater than the scale $i(\infty)^{*}$ of the health food safety scare behavior diffusion in network $K$.

Proof. Theorem 2(1) is untenable. In particular, if the average degree $\left\langle K^{\prime}\right\rangle$ of the health food consumer network $K^{\prime}$ is greater than the average degree $\langle K\rangle$ of the health food consumer network $K$, then $\Theta(\infty)^{* \prime} \leq \Theta(\infty)^{*}$; that is, $\Theta(\infty)^{*} \geq \Theta(\infty)^{* \prime}=G_{k}^{\prime}\left(\Theta(\infty)^{*}\right)$. The equilibrium value $\Theta(\infty)^{*}$ of the health food safety scare behavior diffusion is unique, and $\Theta(\infty)^{*}>0$ when $\lambda_{0}>\lambda_{0} *$. If $H(k)=$ $\lambda_{k} k \Theta(\infty) /\langle k\rangle\left[\lambda_{k} k \Theta(\infty)(1+\mu / \beta)+\mu\right]$, then we can obtain $\partial H(k) / \partial k=\mu \lambda_{k} \Theta(\infty) /\left(\langle k\rangle\left[\lambda_{k} k \Theta(\infty)(1+\mu / \beta)+\mu\right]\right)^{2}>$ 0 . Thus, $H(k)$ is the monotonically increasing function of $k$. According to Jackson [37], if the average degree $\left\langle K^{\prime}\right\rangle$ of network $K^{\prime}$ is greater than the average degree $\langle K\rangle$ of network $K$, then $P^{\prime}$ first-order stochastic dominates $P$. Thus, if the average degree $\left\langle K^{\prime}\right\rangle$ of the health food consumer network $K^{\prime}$ is greater than the average degree $\langle K\rangle$ of the health food consumer network $K$, then $\sum_{k} H(k) P^{\prime}(k)>\sum_{k} H(k) P(k)$. According to (5), $\forall \Theta(\infty)>0, G_{k}^{\prime}(\Theta(\infty))>G_{k}(\Theta(\infty))$ can be obtained. Accordingly, $\forall \Theta(\infty)^{*}>0, G_{k}^{\prime}\left(\Theta(\infty)^{*}\right)>$ $G_{k}\left(\Theta(\infty)^{*}\right)$ and $\Theta(\infty)^{*} \geq G_{k}^{\prime}\left(\Theta(\infty)^{*}\right)>G_{k}\left(\Theta(\infty)^{*}\right)$ can be obtained. This result contradicts $\Theta(\infty)^{*}=G_{k}\left(\Theta(\infty)^{*}\right)$. Thus, our hypothesis is tenable, and Theorem 2(1) is true.

According to Theorem 2(1), $\Theta(\infty)^{* \prime}>\Theta(\infty)^{*}$ when the average degree $\left\langle K^{\prime}\right\rangle$ of the health food consumer network $K^{\prime}$ is greater than the average degree $\langle K\rangle$ of the health food consumer network $K$. Clearly, $i_{k}(\infty)=\lambda_{k} k \Theta(\infty) /$ $\left(\lambda_{k} k \Theta(\infty)(1+\mu / \beta)+\mu\right)$ is the monotonically increasing concave function of $\Theta(\infty)$. Thus, for any $k>0, i_{k}(\infty)^{* \prime}>$ $i_{k}(\infty)^{*}$ and $\sum_{k} i_{k}(\infty)^{* \prime}>\sum_{k} i_{k}(\infty)^{*}$ can be obtained. Furthermore, $\sum_{k} P^{\prime}(k) i_{k}(\infty)^{* \prime}>\sum_{k} P^{\prime}(k) i_{k}(\infty)^{*}$, that is, $i(\infty)^{* \prime}>\sum_{k} P^{\prime}(k) i_{k}(\infty)^{*}$. Apparently, $i_{k}(\infty)=\lambda_{k} k \Theta(\infty) /$ $\left(\lambda_{k} k \Theta(\infty)(1+\mu / \beta)+\mu\right)$ is the monotonically increasing concave function of $k$. Jackson [37] noted that if the average degree $\left\langle K^{\prime}\right\rangle$ of network $K^{\prime}$ is greater than the average degree $\langle K\rangle$ of network $K$, then $P^{\prime}$ first-order stochastic dominates $P$. Therefore, $\sum_{k} P^{\prime}(k) i_{k}(\infty)^{*}>\sum_{k} P(k) i_{k}(\infty)^{*}$ and $i(\infty)^{* \prime}>$ $\sum_{k} P^{\prime}(k) i_{k}(\infty)^{*}>\sum_{k} P(k) i_{k}(\infty)^{*}=i(\infty)^{*}$ can be obtained. Thus, Theorem 2(2) is proven.

Theorem 3. If the heterogeneity of the health food consumer network $K^{\prime}$ is greater than the heterogeneity of the health food consumer network $K$, then the equilibrium value $\Theta(\infty){ }^{* \prime}$ of the health food safety scare behavior diffusion in network $K^{\prime}$ is lower than the equilibrium value $\Theta(\infty)^{*}$ of the health food safety scare behavior diffusion in network $K$ when $\lambda_{0}>\lambda_{0} *$.

Proof. Theorem 3 is untenable. In particular, if the heterogeneity of the health food consumer $K^{\prime}$ is higher than the heterogeneity of the health food consumer network $K$, then $\Theta(\infty)^{*} \leq \Theta(\infty)^{* \prime}=G_{k}^{\prime}\left(\Theta(\infty)^{*}\right)$ can be obtained. Furthermore, $\Theta(\infty)$ has the only equilibrium point in the interval
$[0,1]$ and $\Theta(\infty)^{*}>0 . \partial^{2} H(k) / \partial k^{2}=-2\langle k\rangle \lambda_{k} \Theta(\infty)(1+$ $\mu / \beta) /\left(\langle k\rangle\left[\lambda_{k} k \Theta(\infty)(1+\mu / \beta)+\mu\right]\right)^{3}<0$ is yielded. Thus, $H(k)$ is a concave function of $k$. According to Jackson [37], if the heterogeneity of the network $K^{\prime}$ is greater than the heterogeneity of the network $K$, then $P$ second-order stochastic dominates $P^{\prime}$. Therefore, $\sum_{k} H(k) P(k)>\sum_{k} H(k) P^{\prime}(k)$ can be obtained. According to (5), $\forall \Theta(\infty)>0, G_{k}(\Theta(\infty))>$ $G_{k}^{\prime}(\Theta(\infty))$. Thus, $\forall \Theta(\infty)^{*}>0, G_{k}\left(\Theta(\infty)^{*}\right)>G_{k}^{\prime}\left(\Theta(\infty)^{*}\right)$. Accordingly, $\Theta(\infty)^{*} \leq \Theta(\infty)^{* \prime}=G_{k}^{\prime}\left(\Theta(\infty)^{*}\right)<G_{k}\left(\Theta(\infty)^{*}\right)$. This finding contradicts $\Theta(\infty)^{*}=G_{k}\left(\Theta(\infty)^{*}\right)$. Thus, our hypothesis is tenable, and Theorem 3 is true.

\section{Analogue Simulation}

Numerical simulation analysis is the most effective means of testing real-time dynamic data without the requirement for numerous empirical validations. Therefore, following Chen et al. [3-5] and He et al. [44], we simulate the health food safety scare behavior diffusion by using MATLAB 2016b software.

As the network of health food consumers is a complex network, it is very difficult to obtain the characteristics or the real datasets of the network. Therefore, we selected the most representative three heterogeneous networks to theoretically study the health food safety scare behavior diffusion. BA scale-free network [20], WS network [19], and Exponential network were used. We used BA network, WS network, and Exponential network to describe the feature of different network structure. In fact, fewer nodes have many direct connections with other nodes in BA network, but a large number of nodes have various direct connections with other nodes in WS network. And the heterogeneity of Exponential network is between them.

In the three network models, the degree distribution of BA network is $P(k) \propto 2 m^{2} / k^{3}$, the degree distribution of long distance connection of nodes in WS network is equal to 0.05 , and the degree distribution of Exponential network is $P(k) \propto$ $e^{-\varepsilon k / 2 m}$. Then, let $N=1000$, and let $m_{0}=m=5, \varepsilon=2$.

4.1. Analysis of the Threshold of the Rate of Health Food Safety Scare Behavior Diffusion. We analyze the threshold of the rate of health food safety scare behavior diffusion to depict the evolution characteristics of the health food safety scare behavior diffusion under the influence of the health food safety information transparency and health food consumers' ability to process information.

Figure 5 demonstrates the effects of the health food safety information transparency $\theta$ and the degree $k$ of health food consumers' ability to process information $\eta_{k}$ on the threshold $\lambda_{0} *$ of the rate of health food safety scare behavior diffusion under three network structures. Theorem 1 is verified visually in Figure 5; that is, the threshold $\lambda_{0} *$ of the rate of health food safety scare behavior diffusion is the monotonically increasing function of the health food safety information transparency $\theta$ and the degree $k$ of health food consumers' ability to process information $\eta_{k}$. An increase in the health food safety information transparency $\theta$ and the degree $k$ of health food consumers' ability to process information $\eta_{k}$ increases the threshold $\lambda_{0} *$ of the rate of health food 


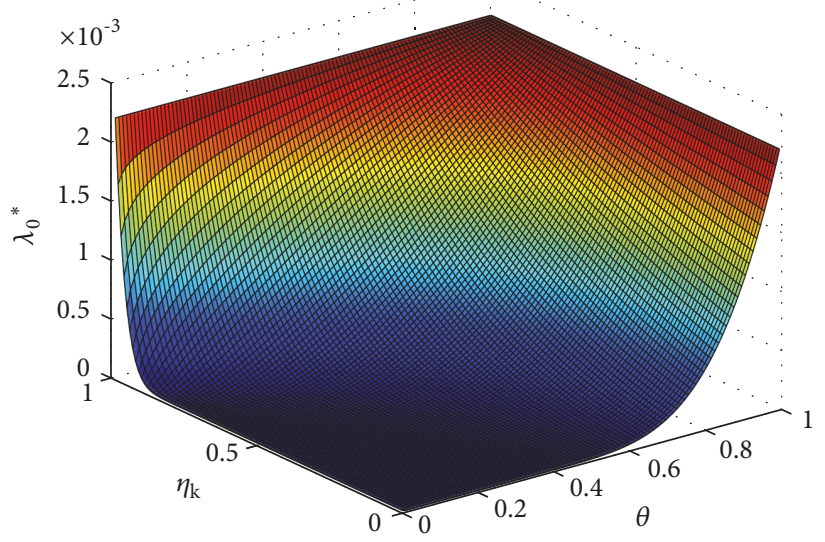

(a) BA network

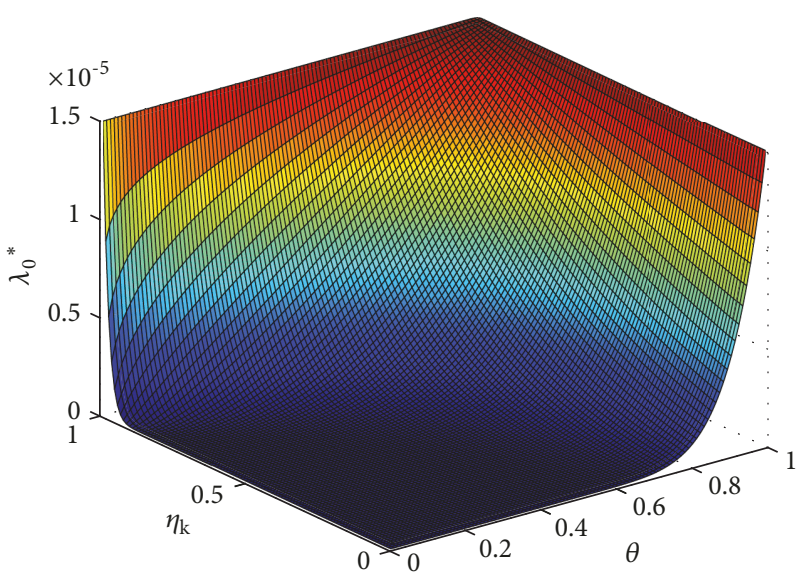

(b) WS network

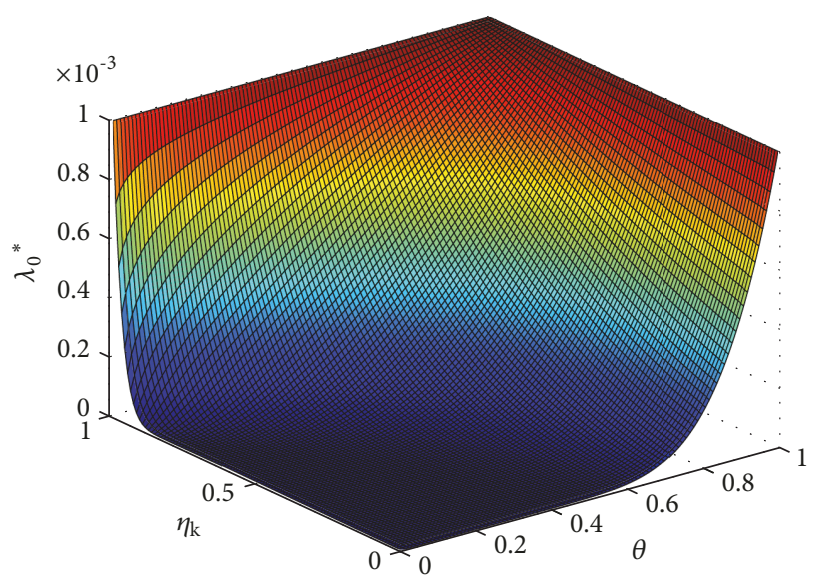

(c) Exponential network

FIGURE 5: Change rules in the threshold of the rate of health food safety scare behavior diffusion under three network structures: (a) BA network, which exerts the effects of the health food safety information transparency $\theta$ and the degree $k$ of health food consumers' ability to process information $\eta_{k}$ on the threshold of the rate of health food safety scare behavior diffusion $\lambda_{0} *$; (b) WS network, which shows the effects of the health food safety information transparency $\theta$ and the degree $k$ of health food consumers' ability to process information $\eta_{k}$ on the threshold of the rate of health food safety scare behavior diffusion $\lambda_{0} *$; (c) Exponential network, which shows the effects of the health food safety information transparency $\theta$ and the degree $k$ of health food consumers' ability to process information $\eta_{k}$ on the threshold of the rate of health food safety scare behavior diffusion $\lambda_{0} *$ (where $k=1000$ and $\mu=0.01$ ).

safety scare behavior diffusion because a high health food safety information transparency indicates comprehensive information on health food in the market for health food consumers. In this scenario, spreading scare behavior when a health food safety incident occurs is difficult. Similarly, a high health food consumers' ability to process information denotes a rational analysis of the health food safety scare behavior. Consequently, no herd behavior will emerge, and health food consumers will inhibit spreading or refuse to spread scare behavior.

A comparison of Figures 5(a), 5(b), and 5(c) indicates that the thresholds $\lambda_{0} *$ of the rate of health food safety scare behavior diffusion differ between the three network structures. The threshold of the rate of health food safety scare behavior diffusion in the WS network is much lower than the threshold of the rate of health food safety scare behavior diffusion in the BA network and the Exponential network; the threshold of the rate of health food safety scare behavior diffusion in the Exponential network is lower than the threshold of the rate of health food safety scare behavior diffusion in the BA network. These results suggest that the health food safety scare behavior in the BA network is more difficult to spread. This phenomenon is attributed to the improved heterogeneity of the BA network. In a network with high heterogeneity, the heterogeneity of the connecting edges of health food consumers and the resistance to and spread of information are also high. Thus, spreading health food safety scare behavior is difficult. This phenomenon also reveals that the structural characteristic of a network significantly affects the health food safety scare behavior diffusion.

To better analyze the threshold of the rate of health food safety scare behavior diffusion to depict the evolution characteristics of the health food safety scare behavior diffusion under the influence of the health food safety information 
TABLE 2: Sensitivity analysis of the health food safety information transparency $\theta$ and the degree $k$ of health food consumers' ability to process information $\eta_{k}$ to the rate of health food safety scare behavior diffusion $\lambda_{0} *$ in the BA network.

\begin{tabular}{|c|c|c|c|c|c|c|c|c|c|c|c|}
\hline \multirow{2}{*}{$\theta$} & \multicolumn{9}{|c|}{$\eta_{k}$} & \multirow{2}{*}{ Expectation } & \multirow{2}{*}{ Variance } \\
\hline & 0.1 & 0.2 & 0.3 & 0.4 & 0.5 & 0.6 & 0.7 & 0.8 & 0.9 & & \\
\hline 0.1 & 4.79E-39 & $2.92 \mathrm{E}-21$ & $5.54 \mathrm{E}-15$ & $9.90 \mathrm{E}-12$ & $9.95 \mathrm{E}-10$ & $2.29 \mathrm{E}-08$ & $2.24 \mathrm{E}-07$ & $1.27 \mathrm{E}-06$ & 4.97E-06 & $7.20 \mathrm{E}-07$ & $2.71 \mathrm{E}-12$ \\
\hline 0.2 & $3.65 \mathrm{E}-31$ & $3.30 \mathrm{E}-18$ & $2.29 \mathrm{E}-13$ & $9.10 \mathrm{E}-11$ & 3.99E-09 & $5.50 \mathrm{E}-08$ & 3.83E-07 & $1.71 \mathrm{E}-06$ & $5.64 \mathrm{E}-06$ & $3.52 \mathrm{E}-12$ & $3.52 \mathrm{E}-12$ \\
\hline 0.3 & $6.64 \mathrm{E}-25$ & $1.31 \mathrm{E}-15$ & $6.23 \mathrm{E}-12$ & $6.89 \mathrm{E}-10$ & $1.46 \mathrm{E}-08$ & $1.27 \mathrm{E}-07$ & $6.42 \mathrm{E}-07$ & $2.29 \mathrm{E}-06$ & $6.41 \mathrm{E}-06$ & $4.59 \mathrm{E}-12$ & $4.59 \mathrm{E}-12$ \\
\hline 0.4 & $6.23 \mathrm{E}-20$ & $2.13 \mathrm{E}-13$ & $1.16 \mathrm{E}-10$ & 4.37E-09 & $4.88 \mathrm{E}-08$ & 2.79E-07 & $1.06 \mathrm{E}-06$ & $3.05 \mathrm{E}-06$ & $7.26 \mathrm{E}-06$ & $6.00 \mathrm{E}-12$ & $6.00 \mathrm{E}-12$ \\
\hline 0.5 & $5.56 \mathrm{E}-16$ & $1.63 \mathrm{E}-11$ & $1.56 \mathrm{E}-09$ & $2.36 \mathrm{E}-08$ & $1.51 \mathrm{E}-07$ & $5.92 \mathrm{E}-07$ & $1.71 \mathrm{E}-06$ & $4.04 \mathrm{E}-06$ & $8.22 \mathrm{E}-06$ & $7.88 \mathrm{E}-12$ & $7.88 \mathrm{E}-12$ \\
\hline 0.6 & $7.63 \mathrm{E}-13$ & $6.55 \mathrm{E}-10$ & $1.55 \mathrm{E}-08$ & $1.10 \mathrm{E}-07$ & $4.31 \mathrm{E}-07$ & $1.21 \mathrm{E}-06$ & 2.73E-06 & 5.31E-06 & $9.29 \mathrm{E}-06$ & $1.04 \mathrm{E}-11$ & $1.04 \mathrm{E}-11$ \\
\hline 0.7 & $2.37 \mathrm{E}-10$ & $1.52 \mathrm{E}-08$ & $1.19 \mathrm{E}-07$ & 4.46E-07 & $1.15 \mathrm{E}-06$ & $2.38 \mathrm{E}-06$ & $4.28 \mathrm{E}-06$ & $6.95 \mathrm{E}-06$ & $1.05 \mathrm{E}-05$ & $1.37 \mathrm{E}-11$ & $1.37 \mathrm{E}-11$ \\
\hline 0.8 & $2.26 \mathrm{E}-08$ & $2.21 \mathrm{E}-07$ & $7.29 \mathrm{E}-07$ & $1.61 \mathrm{E}-06$ & $2.87 \mathrm{E}-06$ & 4.54E-06 & $6.59 \mathrm{E}-06$ & $9.03 \mathrm{E}-06$ & $1.18 \mathrm{E}-05$ & $1.77 \mathrm{E}-11$ & $1.77 \mathrm{E}-11$ \\
\hline 0.9 & $8.45 \mathrm{E}-07$ & $2.16 \mathrm{E}-06$ & $3.62 \mathrm{E}-06$ & $5.16 \mathrm{E}-06$ & $6.76 \mathrm{E}-06$ & 8.38E-06 & $1.00 \mathrm{E}-05$ & $1.17 \mathrm{E}-05$ & $1.33 \mathrm{E}-05$ & $1.86 \mathrm{E}-11$ & $1.86 \mathrm{E}-11$ \\
\hline
\end{tabular}

TABLE 3: Sensitivity analysis of the health food safety information transparency $\theta$ and the degree $k$ of health food consumers' ability to process information $\eta_{k}$ to the rate of health food safety scare behavior diffusion $\lambda_{0} *$ in the WS network.

\begin{tabular}{|c|c|c|c|c|c|c|c|c|c|c|c|}
\hline \multirow{2}{*}{$\theta$} & \multicolumn{9}{|c|}{$\eta_{k}$} & \multirow{2}{*}{ Expectation } & \multirow{2}{*}{ Variance } \\
\hline & 0.1 & 0.2 & 0.3 & 0.4 & 0.5 & 0.6 & 0.7 & 0.8 & 0.9 & & \\
\hline 0.1 & $7.66 \mathrm{E}-22$ & $4.83 \mathrm{E}-12$ & $1.39 \mathrm{E}-08$ & 8.63E-07 & $1.09 \mathrm{E}-05$ & 6.17E-05 & $2.16 \mathrm{E}-04$ & 5.63E-04 & $1.19 \mathrm{E}-03$ & $2.28 \mathrm{E}-04$ & $1.66 \mathrm{E}-07$ \\
\hline 0.2 & $1.69 \mathrm{E}-17$ & $2.33 \mathrm{E}-10$ & $1.08 \mathrm{E}-07$ & $2.93 \mathrm{E}-06$ & $2.35 \mathrm{E}-05$ & 9.99E-05 & 2.91E-04 & $6.64 \mathrm{E}-04$ & $1.28 \mathrm{E}-03$ & 2.63E-04 & $1.95 \mathrm{E}-07$ \\
\hline 0.3 & $4.75 \mathrm{E}-14$ & $6.29 \mathrm{E}-09$ & $6.69 \mathrm{E}-07$ & 8.94E-06 & $4.80 \mathrm{E}-05$ & $1.58 \mathrm{E}-04$ & $3.87 \mathrm{E}-04$ & $7.80 \mathrm{E}-04$ & $1.37 \mathrm{E}-03$ & $3.06 \mathrm{E}-04$ & $2.29 \mathrm{E}-07$ \\
\hline 0.4 & $2.61 \mathrm{E}-11$ & $1.04 \mathrm{E}-07$ & $3.35 \mathrm{E}-06$ & $2.47 \mathrm{E}-05$ & $9.35 \mathrm{E}-05$ & $2.44 \mathrm{E}-04$ & $5.10 \mathrm{E}-04$ & $9.14 \mathrm{E}-04$ & $1.47 \mathrm{E}-03$ & 3.63E-04 & $2.69 \mathrm{E}-07$ \\
\hline 0.5 & 3.92E-09 & $1.14 \mathrm{E}-06$ & $1.40 \mathrm{E}-05$ & $6.26 \mathrm{E}-05$ & $1.74 \mathrm{E}-04$ & $3.70 \mathrm{E}-04$ & $6.65 \mathrm{E}-04$ & $1.07 \mathrm{E}-03$ & $1.58 \mathrm{E}-03$ & $4.37 \mathrm{E}-04$ & $3.15 \mathrm{E}-07$ \\
\hline 0.6 & $2.10 \mathrm{E}-07$ & $8.70 \mathrm{E}-06$ & $4.98 \mathrm{E}-05$ & $1.46 \mathrm{E}-04$ & $3.11 \mathrm{E}-04$ & $5.48 \mathrm{E}-04$ & 8.59E-04 & $1.24 \mathrm{E}-03$ & $1.69 \mathrm{E}-03$ & $5.39 \mathrm{E}-04$ & $3.66 \mathrm{E}-07$ \\
\hline 0.7 & 4.97E-06 & 4.92E-05 & $1.53 \mathrm{E}-04$ & $3.17 \mathrm{E}-04$ & $5.34 \mathrm{E}-04$ & 7.97E-04 & $1.10 \mathrm{E}-03$ & $1.44 \mathrm{E}-03$ & $1.80 \mathrm{E}-03$ & $6.88 \mathrm{E}-04$ & 4.14E-07 \\
\hline 0.8 & $6.12 \mathrm{E}-05$ & $2.15 \mathrm{E}-04$ & $4.15 \mathrm{E}-04$ & $6.41 \mathrm{E}-04$ & 8.84E-04 & $1.14 \mathrm{E}-03$ & $1.40 \mathrm{E}-03$ & $1.66 \mathrm{E}-03$ & $1.93 \mathrm{E}-03$ & $9.26 \mathrm{E}-04$ & $4.26 \mathrm{E}-07$ \\
\hline 0.9 & $4.50 \mathrm{E}-04$ & $7.54 \mathrm{E}-04$ & $1.00 \mathrm{E}-03$ & $1.22 \mathrm{E}-03$ & $1.42 \mathrm{E}-03$ & $1.59 \mathrm{E}-03$ & $1.76 \mathrm{E}-03$ & $1.91 \mathrm{E}-03$ & $2.06 \mathrm{E}-03$ & $1.35 \mathrm{E}-03$ & $2.94 \mathrm{E}-07$ \\
\hline
\end{tabular}

TABLE 4: Sensitivity analysis of the health food safety information transparency $\theta$ and the degree $k$ of health food consumers' ability to process information $\eta_{k}$ to the rate of health food safety scare behavior diffusion $\lambda_{0} *$ in the Exponential network.

\begin{tabular}{|c|c|c|c|c|c|c|c|c|c|c|c|}
\hline \multirow{2}{*}{$\theta$} & \multicolumn{9}{|c|}{$\eta_{k}$} & \multirow{2}{*}{ Expectation } & \multirow{2}{*}{ Variance } \\
\hline & 0.1 & 0.2 & 0.3 & 0.4 & 0.5 & 0.6 & 0.7 & 0.8 & 0.9 & & \\
\hline 0.1 & $3.95 \mathrm{E}-22$ & $9.16 \mathrm{E}-13$ & 3.08E-09 & $2.31 \mathrm{E}-07$ & $3.43 \mathrm{E}-06$ & $2.19 \mathrm{E}-05$ & $8.49 \mathrm{E}-05$ & $2.40 \mathrm{E}-04$ & $5.44 \mathrm{E}-04$ & 9.94E-05 & $3.40 \mathrm{E}-08$ \\
\hline 0.2 & $6.85 \mathrm{E}-18$ & $5.03 \mathrm{E}-11$ & $2.69 \mathrm{E}-08$ & $8.50 \mathrm{E}-07$ & $7.78 \mathrm{E}-06$ & 3.67E-05 & $1.16 \mathrm{E}-04$ & 2.85E-04 & $5.84 \mathrm{E}-04$ & $1.15 \mathrm{E}-04$ & $3.99 \mathrm{E}-08$ \\
\hline 0.3 & $1.72 \mathrm{E}-14$ & $1.55 \mathrm{E}-09$ & $1.84 \mathrm{E}-07$ & $2.80 \mathrm{E}-06$ & $1.67 \mathrm{E}-05$ & $6.01 \mathrm{E}-05$ & $1.58 \mathrm{E}-04$ & $3.38 \mathrm{E}-04$ & $6.26 \mathrm{E}-04$ & $1.34 \mathrm{E}-04$ & $4.68 \mathrm{E}-08$ \\
\hline 0.4 & $9.15 \mathrm{E}-12$ & $2.90 \mathrm{E}-08$ & $1.02 \mathrm{E}-06$ & $8.33 \mathrm{E}-06$ & $3.42 \mathrm{E}-05$ & $9.60 \mathrm{E}-05$ & $2.12 \mathrm{E}-04$ & 3.99E-04 & $6.71 \mathrm{E}-04$ & $1.58 \mathrm{E}-04$ & $5.51 \mathrm{E}-08$ \\
\hline 0.5 & $1.41 \mathrm{E}-09$ & $3.56 \mathrm{E}-07$ & $4.70 \mathrm{E}-06$ & $2.26 \mathrm{E}-05$ & 6.69E-05 & $1.50 \mathrm{E}-04$ & $2.82 \mathrm{E}-04$ & $4.70 \mathrm{E}-04$ & 7.19E-04 & $1.91 \mathrm{E}-04$ & $6.47 \mathrm{E}-08$ \\
\hline 0.6 & 7.96E-08 & $3.04 \mathrm{E}-06$ & $1.82 \mathrm{E}-05$ & 5.63E-05 & $1.25 \mathrm{E}-04$ & 2.29E-04 & $3.71 \mathrm{E}-04$ & $5.51 \mathrm{E}-04$ & $7.69 \mathrm{E}-04$ & $2.36 \mathrm{E}-04$ & 7.55E-08 \\
\hline 0.7 & $2.03 \mathrm{E}-06$ & $1.91 \mathrm{E}-05$ & $6.09 \mathrm{E}-05$ & $1.30 \mathrm{E}-04$ & $2.25 \mathrm{E}-04$ & $3.44 \mathrm{E}-04$ & $4.84 \mathrm{E}-04$ & $6.45 \mathrm{E}-04$ & 8.23E-04 & $3.04 \mathrm{E}-04$ & $8.60 \mathrm{E}-08$ \\
\hline 0.8 & 2.72E-05 & $9.17 \mathrm{E}-05$ & $1.78 \mathrm{E}-04$ & $2.78 \mathrm{E}-04$ & $3.88 \mathrm{E}-04$ & 5.05E-04 & $6.26 \mathrm{E}-04$ & $7.52 \mathrm{E}-04$ & $8.80 \mathrm{E}-04$ & $4.14 \mathrm{E}-04$ & $8.90 \mathrm{E}-08$ \\
\hline 0.9 & $2.18 \mathrm{E}-04$ & $3.52 \mathrm{E}-04$ & $4.62 \mathrm{E}-04$ & $5.59 \mathrm{E}-04$ & $6.47 \mathrm{E}-04$ & $7.28 \mathrm{E}-04$ & 8.03E-04 & $8.73 \mathrm{E}-04$ & $9.40 \mathrm{E}-04$ & $6.20 \mathrm{E}-04$ & $5.93 \mathrm{E}-08$ \\
\hline
\end{tabular}

transparency and health food consumers' ability to process information, we make the sensitivity analysis within the three networks. The results are shown as in Tables 2, 3, and 4.

The sensitivity analysis results in Tables 2,3 , and 4 show that the threshold $\lambda_{0}$ * of the rate of health food safety scare behavior diffusion is the monotonically increasing function of the health food safety information transparency $\theta$ and the degree $k$ of health food consumers' ability to process information $\eta_{k}$. Furthermore, the health food safety information transparency $\theta$ shows the characteristics of incremental margins in relation to the threshold $\lambda_{0} *$ of the rate of health food safety scare behavior diffusion, whereas the degree $k$ of health food consumers' ability to process information $\eta_{k}$ denotes the characteristics of diminishing margins in relation to the threshold $\lambda_{0}$ * of the rate of health food safety scare behavior diffusion. 


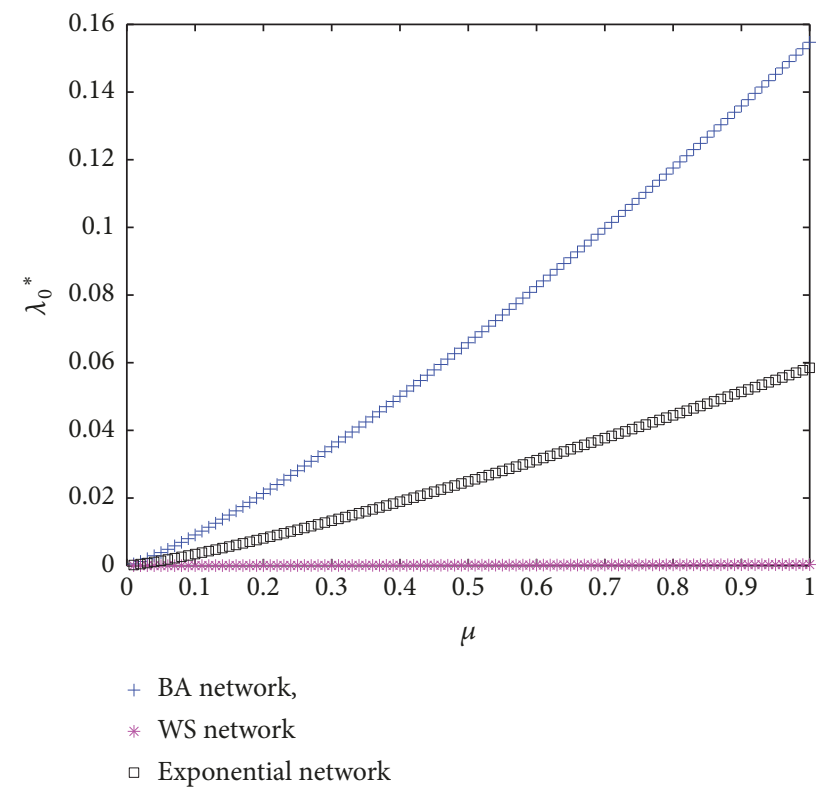

FIGURE 6: Effects of the recovery rate $\mu$ of health food consumers on the threshold $\lambda_{0} *$ of the rate of health food safety scare behavior diffusion under three network structures (where $\theta=0.5, \eta_{k}=0.7$, and $k=1000)$.

Then, we simulate the relationship between the recovery rate $\mu$ and the threshold $\lambda_{0} *$ of the rate of health food safety scare behavior diffusion. The result is shown as in Figure 6.

Figure 6 displays the effects of the recovery rate $\mu$ of health food consumers on the threshold $\lambda_{0} *$ of the rate of health food safety scare behavior diffusion under three network structures. Figure 6 verifies the conclusion of Theorem 1, that is, the threshold $\lambda_{0} *$ of the rate of health food safety scare behavior diffusion is the monotonically increasing function of the recovery rate $\mu$ of health food consumers. In particular, the threshold $\lambda_{0} *$ of the rate of health food safety scare behavior diffusion increases with the recovery rate $\mu$ of health food consumers, and the rate is low. This outcome is due to a high recovery rate of health food consumers indicates their improved ability to resist scare behavior. Thus, the threshold required to achieve the health food safety scare behavior diffusion must be high. In addition, Figure 6 illustrates that the threshold of the rate of health food safety scare behavior diffusion is much lower in the WS network than in the BA network and Exponential network. Furthermore, the former tends to zero. And the change trend in the Exponential network is much smaller in the BA network, while which in the WS network is the smallest.

4.2. Analysis of the Scale of the Health Food Safety Scare Behavior Diffusion. Subsequently, we analyze the scale of the health food safety scare behavior diffusion. The results are shown as in Figure 7.

Figure 7 presents the effects of the network structure of health food consumers, the health food safety information transparency $\theta$, the degree $k$ of health food consumers' ability to process information $\eta_{k}$, and the degree $k$ of health food consumers on the scale $i(\infty)$ of the health food safety scare behavior diffusion. Figures 7(a)-7(f) demonstrate that a high heterogeneity of a health food consumer network denotes a low scale of health food safety scare behavior diffusion.

In Figures 7(a) and 7(c), with increasing in the health food safety information transparency $\theta$, the scale $i(\infty)$ of the health food safety scare behavior diffusion decreases. That is, health food safety information transparency exerts inhibitory effects on health food safety scare behavior diffusion and shows the characteristics of incremental margins. In addition, the inhibitory effect of health food safety information transparency on health food safety scare behavior diffusion is apparent in the BA network because health food consumers exhibit few direct edges in the BA network, and a low health food safety information transparency can exert a strong inhibitory effect on the health food safety scare behavior diffusion. By contrast, in the WS network, the direct edges of health food consumers are relatively large, and their similarity is high; moreover, a low health food safety information transparency can hardly exert a strong inhibitory effect on the health food safety scare behavior diffusion. As for the Exponential network, the situation is between the BA network and WS network.

In Figures $7(\mathrm{~b})$ and $7(\mathrm{~d})$, the scale $i(\infty)$ of the health food safety scare behavior diffusion decreases with an increase in the degree $k$ of health food consumers' ability to process information $\eta_{k}$. That is, the degree $k$ of health food consumers' ability to process information $\eta_{k}$ exerts an inhibitory effect on the health food safety scare behavior diffusion and shows the characteristics of incremental margins. Similarly, a high health food consumers' ability to process information denotes a strong inhibitory effect on the health food safety scare behavior diffusion.

In Figures 7(e) and 7(f), the degree $k$ of health food consumers indicates a marginal diminishing rising characteristic in relation to the scale $i(\infty)$ of the health food safety scare behavior diffusion. Moreover, the scales of the health food safety scare behavior diffusion under different networks tend to a steady value with an increase in the degree $k$ of health food consumers.

Last, we simulate the relationship between the recovery rate $\mu$ and the scale $i(\infty)$ of the health food safety scare behavior diffusion. The result is shown as in Figure 8.

Figure 8 illustrates the effects of the recovery rate $\mu$ of health food consumers on the scale $i(\infty)$ of the health food safety scare behavior diffusion under three kinds of network structures. The scale $i(\infty)$ of the health food safety scare behavior diffusion decreases and shows the characteristics of diminishing margins with the increase in the recovery rate $\mu$ of health food consumers. Figure 8 also demonstrates that a high heterogeneity of health food consumer networks indicates a low scale of the health food safety scare behavior diffusion.

\section{Conclusion}

The health food safety scare behavior diffusion is influenced by various factors. On the basis of the concept of complex network, this study extends the existing epidemic SIRS 

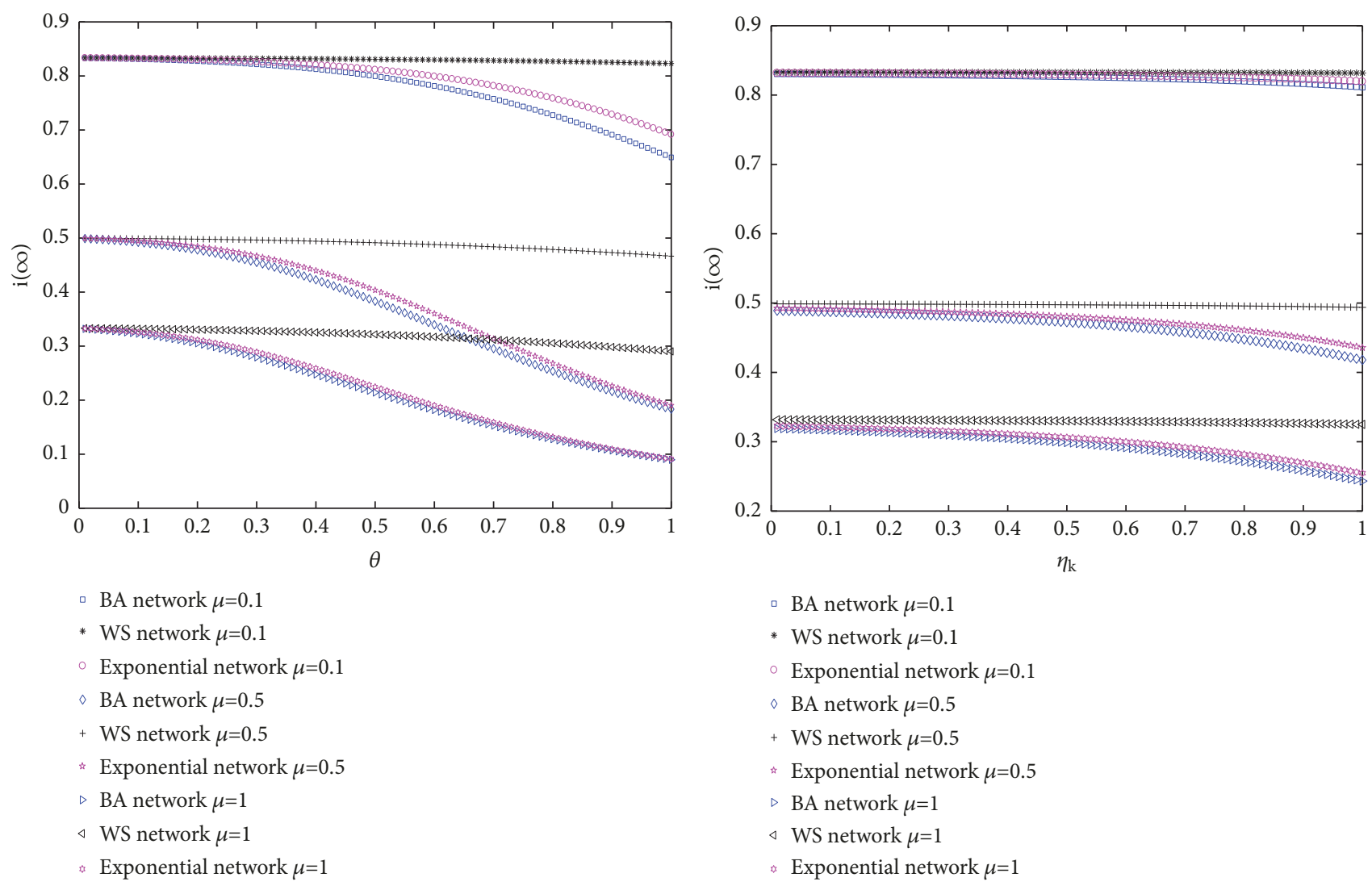

(a)

(b)
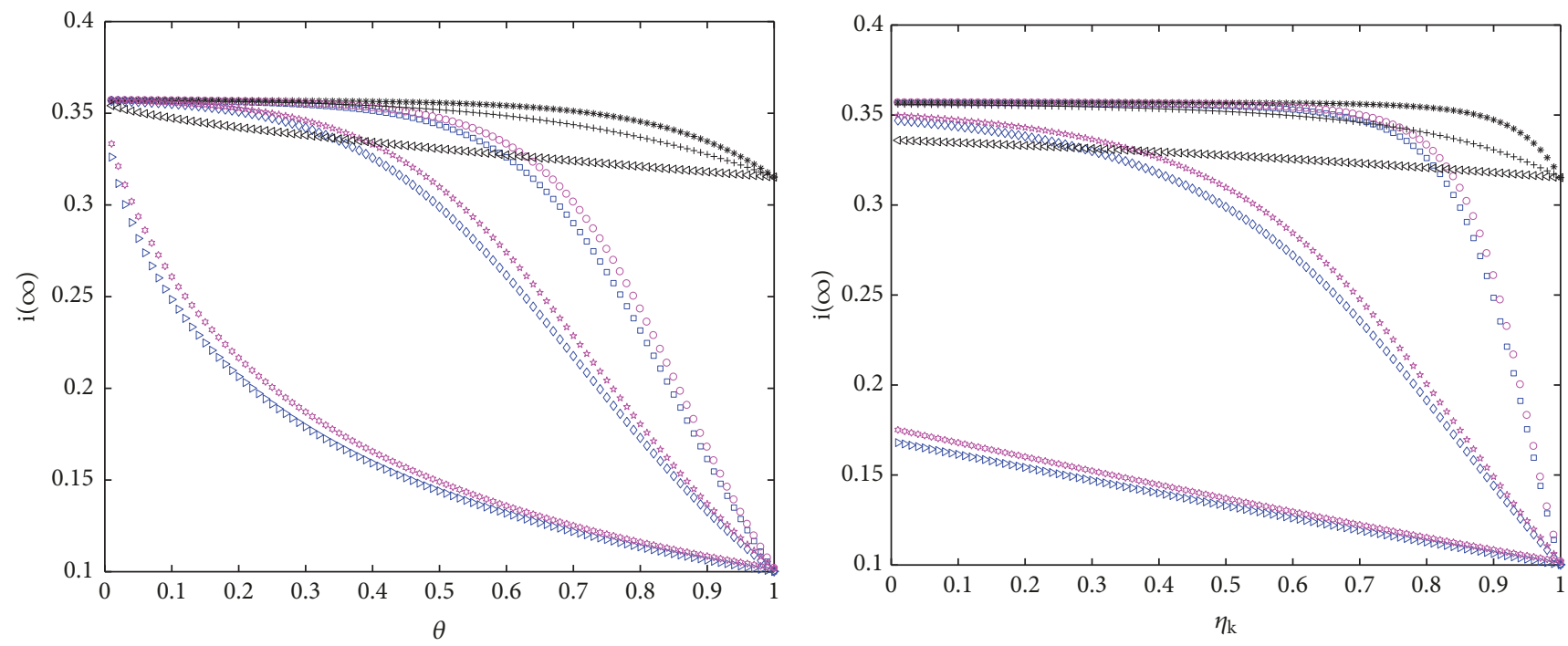

- BA network $\eta_{\mathrm{k}}=0.1$

- BA network $\theta=0.1$

* WS network $\eta_{\mathrm{k}}=0.1$

* WS network $\theta=0.1$

- Exponential network $\eta_{\mathrm{k}}=0.1$

$\diamond$ BA network $\eta_{\mathrm{k}}=0.5$

- Exponential network $\theta=0.1$

$\diamond$ BA network $\theta=0.5$

+ WS network $\theta=0.5$

म Exponential network $\theta=0.5$

$\triangleright$ BA network $\theta=0.9$

$\triangleleft$ WS network $\theta=0.9$

* Exponential network $\theta=0.9$

(c)

Figure 7: Continued. 

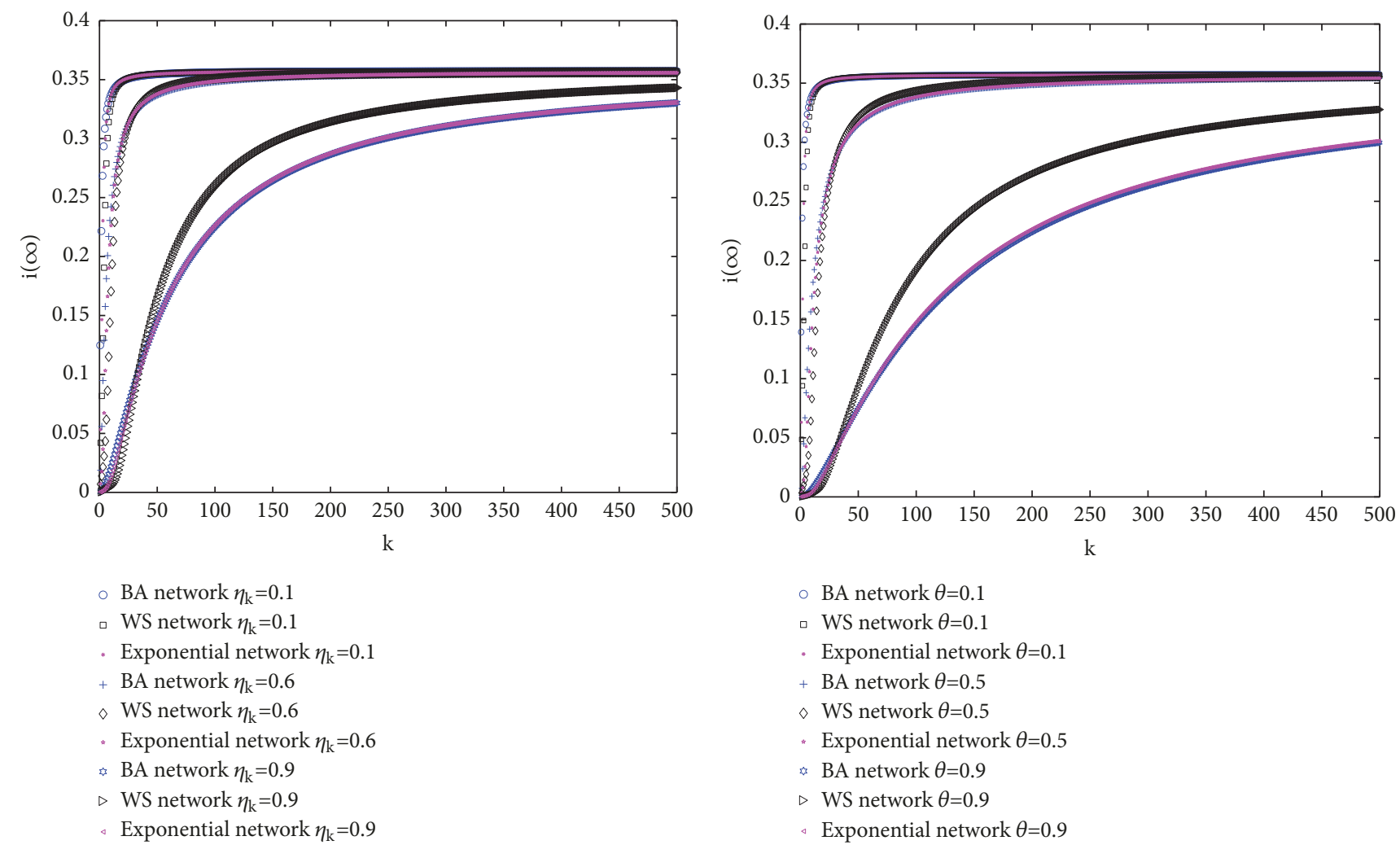

(e)

FIGURE 7: Change rules of the scale $i(\infty)$ of the health food safety scare behavior diffusion. (a) Effects of the health food safety information transparency $\theta$ on $i(\infty)$ under different network structures and recovery rates $\mu$ (where $\lambda_{0}=0.001, \beta=0.5, \eta_{k}=0.7$, and $k=1000$ ). (b) Effects of the degree $k$ of health food consumers' ability to process information $\eta_{k}$ on $i(\infty)$ under different network structures and recovery rates $\mu$ (where $\lambda_{0}=0.005, \beta=0.5, \theta=0.7$, and $k=1000$ ). (c) Effects of the health food safety information transparency $\theta$ on $i(\infty)$ under different network structures and degree $k$ of health food consumers' ability to process information $\eta_{k}$ (where $\lambda_{0}=0.001, \beta=0.5, \mu=0.9$, and $k=1000$ ). (d) Effects of the degree $k$ of health food consumers' ability to process information $\eta_{k}$ on $i(\infty)$ under different network structures and health food safety information transparency $\theta$ (where $\lambda_{0}=0.001, \beta=0.5, \mu=0.9$, and $k=1000$ ). (e) Effects of the degree $k$ of health food consumers on $i(\infty)$ under different network structures and degree $k$ of health food consumers' ability to process information $\eta_{k}$ (where $\lambda_{0}=0.001, \beta=0.5, \mu=0.9$, and $\theta=0.1$ ). (f) Effects of the degree $k$ of health food consumers on $i(\infty)$ under different network structures and health food safety information transparency $\theta$ (where $\lambda_{0}=0.001, \beta=0.5, \mu=0.9$, and $\eta_{k}=0.01$ ).

model and establishes a heterogeneous model of the rate of health food safety scare behavior diffusion by considering health food safety information transparency and health food consumers' ability to process information. The health food safety scare behavior diffusion and its influencing factors are analyzed theoretically using mean-field and network stochastic dominance theories. Mathematical simulation is performed to explore the effects of health food safety information transparency $\theta$, health food consumers' ability to process information $\eta_{k}$, and heterogeneity of health food consumers' networks on the change rules of the threshold $\lambda_{0} *$ of the rate and scale $i(\infty)$ of health food safety scare behavior diffusion. From the results, we have drawn the following main conclusions:

(1) Increases in the health food safety information transparency, health food consumers' ability to process information, and recovery rate of health food consumers can increase the threshold of the rate of health food safety scare behavior diffusion. The health food safety information transparency and recovery rate of health food consumers show marginal incremental rising characteristics in relation to the rate of health food safety scare behavior diffusion, whereas health food consumers' ability to process information reflects a marginal diminishing rising characteristic in relation to the rate of health food safety scare behavior diffusion.

(2) Increases in the health food safety information transparency, health food consumers' ability to process information, and recovery rate of health food consumers can decrease the scale of the health food safety scare behavior diffusion. The health food safety information transparency displays a marginal diminishing decreasing characteristic in relation to the scale of health food safety scare behavior diffusion, whereas the health food consumers' ability to process information and the recovery rate of the health food consumers indicate marginal incremental decreasing characteristics in relation to the scale of the health food safety scare behavior diffusion. 


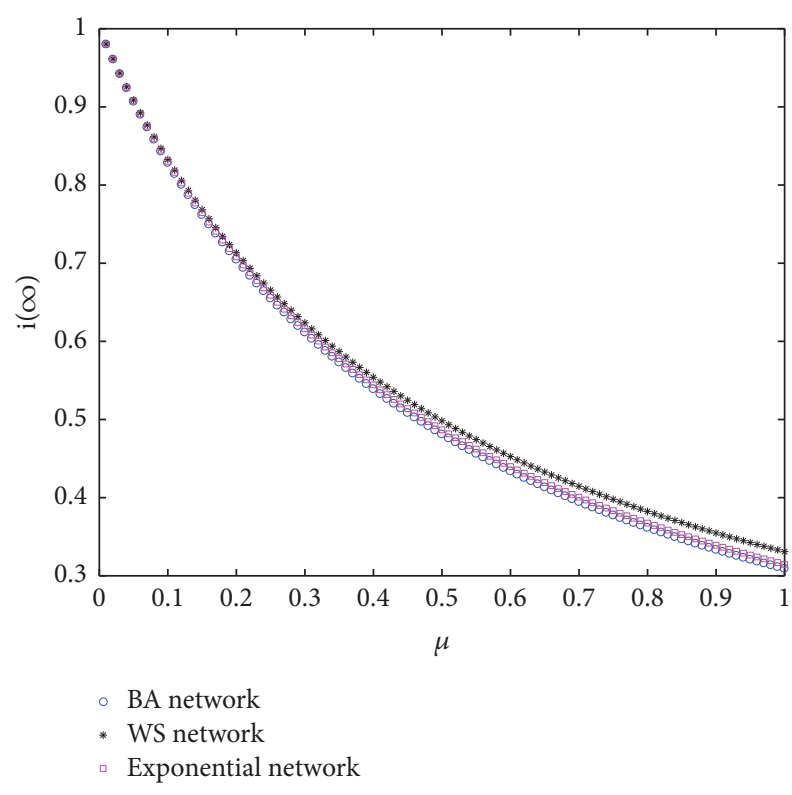

FIGURE 8: Effects of the recovery rate $\mu$ of health food consumers on the scale $i(\infty)$ of the health food safety scare behavior diffusion under three kinds of network structures (where $\lambda_{0}=0.005, \beta=0.5$, $\theta=0.75, \eta_{k}=0.15$, and $k=1000$ ).

(3) The network structure of health food consumers significantly affects the health food safety scare behavior diffusion. A high heterogeneity of the health food consumer network denotes a high threshold of the rate of health food safety scare behavior diffusion and a low diffusion scale.

These conclusions are theoretically and practically crucial to explaining the health food safety scare behavior diffusion. The information processing capacity of health food consumers can be improved by increasing the health food information transparency and guiding these consumers in increasing their health food knowledge. Effective recovery measures after health food safety accidents must be performed, and the structure of the health food consumer network must be changed to manage and control the health food safety scare behavior diffusion.

\section{Data Availability}

The method in this article is computer mathematical simulation. Numerical simulation analysis is the most effective way to test real-time dynamic data without a large number of empirical validations. The authors simulate to explore the characteristics and laws of the evolution of health food safety scare behavior diffusion by using Matlab2016b software. This paper does not have the data that can be obtained because they directly use the plot function of Matlab2016b software to make the images.

\section{Conflicts of Interest}

The authors declare that they have no conflicts of interest.

\section{Acknowledgments}

This work was supported by the Social Science Foundation of Jiangsu Province (no. 18GLC011), the Key Project of Applied Research of Social Science in Jiangsu Province (no. 18SYA042), and the National Natural Science Foundation of China (nos. 71871115 and 71501094).

\section{References}

[1] R. Buchanan, "Understanding and Managing Food Safety Risks," Journal of Food Safety, vol. 16, no. 6, pp. 24-31, 2010.

[2] N. E. Piggott and T. L. Marsh, "Does food safety information impact U.S. meat demand?" American Journal of Agricultural Economics, vol. 86, no. 1, pp. 154-174, 2011.

[3] T. Chen, B. Ma, and J. Wang, "SIRS contagion model of food safety risk," Journal of Food Safety, p. e12410, 2017.

[4] T. Chen, L. Wang, J. Wang, and Q. Yang, "A Network Diffusion Model of Food Safety Scare Behavior considering Information Transparency," Complexity, vol. 2017, Article ID 5724925, 16 pages, 2017.

[5] T. Chen, L. Wang, and J. Wang, "Transparent assessment of the supervision information in China's food safety: A fuzzy-ANP comprehensive evaluation method," Journal of Food Quality, vol. 2017, Article ID 4340869, 14 pages, 2017.

[6] T. Li, J. C. Bernard, Z. A. Johnston, K. D. Messer, and H. M. Kaiser, "Consumer preferences before and after a food safety scare: An experimental analysis of the 2010 egg recall," Food Policy, vol. 66, pp. 25-34, 2017.

[7] D. Smith and P. Riethmuller, "Consumer concerns about food safety in Australia and Japan," International Journal of Social Economics, vol. 26, no. 6, pp. 724-742, 1999.

[8] J. M. E. Pennings, B. Wansink, and M. T. G. Meulenberg, "A note on modeling consumer reactions to a crisis: The case of the mad cow disease," International Journal of Research in Marketing, vol. 19, no. 1, pp. 91-100, 2002.

[9] M. L. Roehm and A. M. Tybout, "When will a brand scandal spill over, and how should competitors respond?" Journal of Marketing Research, vol. 43, no. 3, pp. 366-373, 2006.

[10] M. S. Park, H. N. Kim, and G. J. Bahk, "The analysis of food safety incidents in South Korea, 1998-2016," Food Control, vol. 81, pp. 196-199, 2017.

[11] N. Tanemura, N. Hamadate, and H. Urushihara, "The need for consumer science and regulatory science research on functional foods with health claims - What should we do to harmonize science and technology with society?" Trends in Food Science \& Technology, vol. 67, pp. 280-283, 2017.

[12] J. A. Caswell and E. M. Mojduszka, "Using informational labeling to influence the market for quality in food products," American Journal of Agricultural Economics, vol. 78, no. 5, pp. 1248-1253, 1996.

[13] A. J. M. Beulens, D.-F. Broens, P. Folstar, and G. J. Hofstede, "Food safety and transparency in food chains and networks. Relationships and challenges," Food Control, vol. 16, no. 6, pp. 481-486, 2005.

[14] M. Mazzocchi, A. Lobb, W. B. Traill, and A. Cavicchi, "Food scares and trust: A European study," Journal of Agricultural Economics, vol. 59, no. 1, pp. 2-24, 2008.

[15] S. Cope, L. J. Frewer, J. Houghton, G. Rowe, A. R. H. Fischer, and J. de Jonge, "Consumer perceptions of best practice in food risk 
communication and management: Implications for risk analysis policy," Food Policy, vol. 35, no. 4, pp. 349-357, 2010.

[16] A. P. J. Mol, "Governing China's food quality through transparency: a review," Food Control, vol. 43, no. 6, pp. 49-56, 2014.

[17] W. McKelvey, M. R. Wong, and B. Matis, "Letter Grading and Transparency Promote Restaurant Food Safety in New York City," Journal of Environmental Health, vol. 78, no. 2, pp. 46-48, 2015.

[18] A. Papadopoulos, J. M. Sargeant, S. E. Majowicz et al., "Enhancing public trust in the food safety regulatory system," Health Policy, vol. 107, no. 1, pp. 98-103, 2012.

[19] D. J. Watts and S. H. Strogatz, "Collective dynamics of 'smallworld” networks," Nature, vol. 393, no. 6684, pp. 440-442, 1998.

[20] A. Barabasi and R. Albert, "Emergence of scaling in random networks," Science, vol. 286, no. 5439, pp. 509-512, 1999.

[21] M. E. J. Newman, C. Moore, and D. J. Watts, "Mean-field solution of the small-world network model," Physical Review Letters, vol. 84, no. 14, pp. 3201-3204, 2000.

[22] K. Klemm, M. Á. Serrano, V. M. Eguíluz, and M. S. Miguel, "A measure of individual role in collective dynamics," Scientific Reports, vol. 2, p. 292, 2012.

[23] K. Musial, P. Bródka, and P. De Meo, "Analysis and applications of complex social networks," Complexity, vol. 2017, Article ID 3014163, 2 pages, 2017.

[24] P. Bródka, A. Chmiel, M. Magnani, and G. Ragozini, "Quantifying layer similarity in multiplex networks: a systematic study," Royal Society Open Science, vol. 5, no. 8, p. 171747, 2018.

[25] S. Gu, J. Johnson, F. E. Faisal, and T. Milenković, "From homogeneous to heterogeneous network alignment via colored graphlets," Scientific Reports, vol. 8, no. 1, 2018.

[26] A. Sharma, M. Kitsak, M. H. Cho et al., "Integration of Molecular Interactome and Targeted Interaction Analysis to Identify a COPD Disease Network Module," Scientific Reports, vol. 8, no. 1, 2018.

[27] S. E. Kessler, T. R. Bonnell, J. M. Setchell, and C. A. Chapman, "Social Structure Facilitated the Evolution of Care-giving as a Strategy for Disease Control in the Human Lineage," Scientific Reports, vol. 8, no. 1, 2018.

[28] J. de Jonge, L. Frewer, H. Van Trijp, R. Jan Renes, W. de Wit, and J. Timmers, "Monitoring consumer confidence in food safety: An exploratory study," British Food Journal, vol. 106, pp. 837849, 2004.

[29] P. J. Williamson, "Sales and Service Strategy for the Single European Market," Business Strategy Review, vol. 3, no. 2, pp. 17-43, 2010.

[30] L. Austin, B. F. Liu, and Y. Jin, "How Audiences Seek Out Crisis Information: Exploring the Social-Mediated Crisis Communication Model," Journal of Applied Communication Research, vol. 40, no. 2, pp. 188-207, 2012.

[31] J. P. Quirk and R. Saposnik, "Admissibility and measurable utility functions," Review of Economic Studies, vol. 29, no. 2, pp. 140-146, 1962.

[32] J. Karamata, "Über einen Satz von Vijayaraghavan," Mathematische Zeitschrift, vol. 34, no. 1, pp. 737-740, 1932.

[33] J. Hadar and W. R. Russell, "Rules for ordering uncertain prospects," American Economic Review, vol. 59, pp. 25-34, 1969.

[34] G. Hanoch and H. Levy, "The efficiency analysis of choices involving risk," Review of Economic Studies, vol. 36, no. 3, pp. 335-346, 1969.

[35] M. Rothschild and J. E. Stiglitz, "Increasing risk. I. A definition," Journal of Economic Theory, vol. 2, pp. 225-243, 1970.
[36] P. C. Fishburn, "Convex stochastic dominance with continuous distribution functions," Journal of Economic Theory, vol. 7, no. 2, pp. 143-158, 1976.

[37] M. O. Jackson, The Economics of Social Networks, Cambridge University Press, Cambridge, UK, 2006.

[38] Y.-T. Bian, L. Xu, J.-S. Li, and X.-Q. Liu, "Dynamical evolution of trading behavior on anti-coordination game in complex networks," China Finance Review International, vol. 6, no. 4, pp. 367-379, 2016.

[39] V. Colizza and A. Vespignani, "Epidemic modeling in metapopulation systems with heterogeneous coupling pattern: theory and simulations," Journal of Theoretical Biology, vol. 251, no. 3, pp. 450-467, 2007.

[40] F. Zhang, L. I. Lu, and X. H. Yu, "Survey of transmission models of infectious diseases," System Engineering Theory \& Practice, vol. 31, no. 9, pp. 1736-1744, 2011.

[41] S. Li and Z. Jin, "Dynamic modeling and analysis of sexually transmitted diseases on heterogeneous networks," Physica A: Statistical Mechanics and its Applications, vol. 427, pp. 192-201, 2015.

[42] R. Pastor-Satorras and A. Vespignani, "Epidemics and immunization in scale-free networks," in Handbook of Graph \& Networks, S. ornholdt and H. G. Schuster, Eds., pp. 111-130, 2002.

[43] R. E. Lofstedt, "How can we make food risk communication better: Where are we and where are we going?" Journal of Risk Research, vol. 9, no. 8, pp. 869-890, 2006.

[44] J. He, X. Sui, and S. Li, "An endogenous model of the credit network," Physica A: Statistical Mechanics and its Applications, vol. 441, pp. 1-14, 2016. 


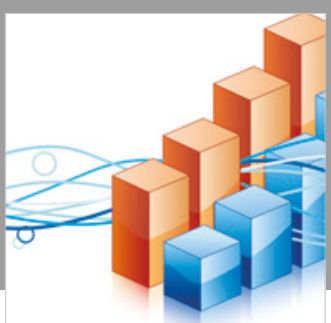

Advances in

Operations Research

\section{-n-m}
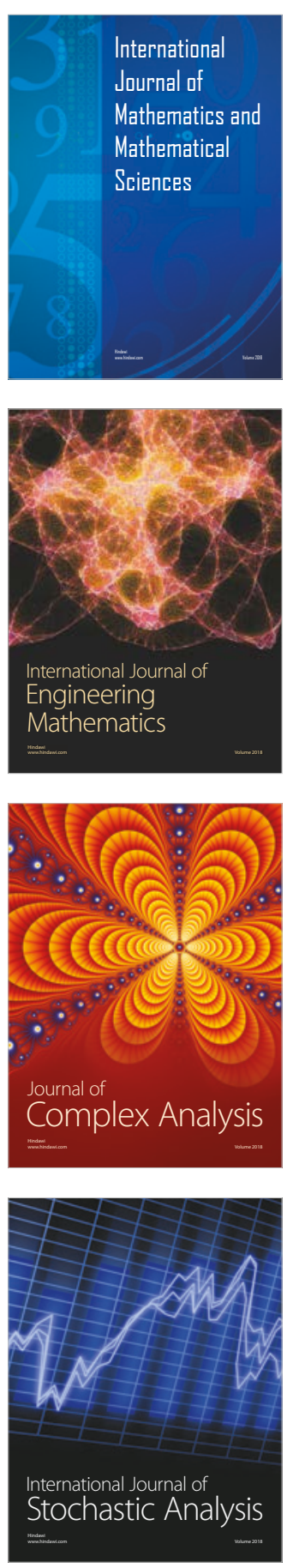
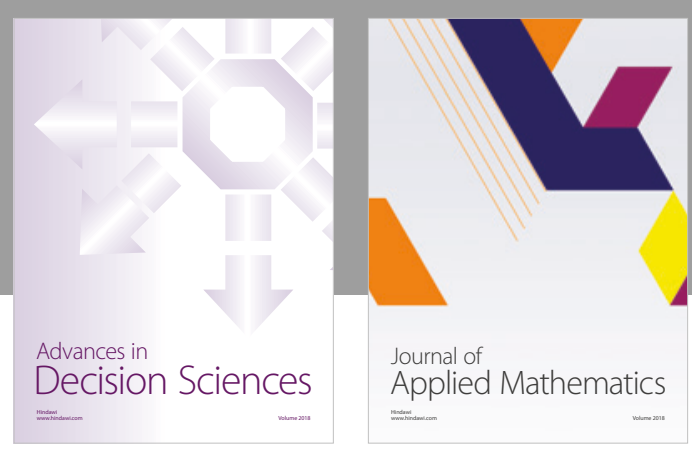

Journal of

Applied Mathematics
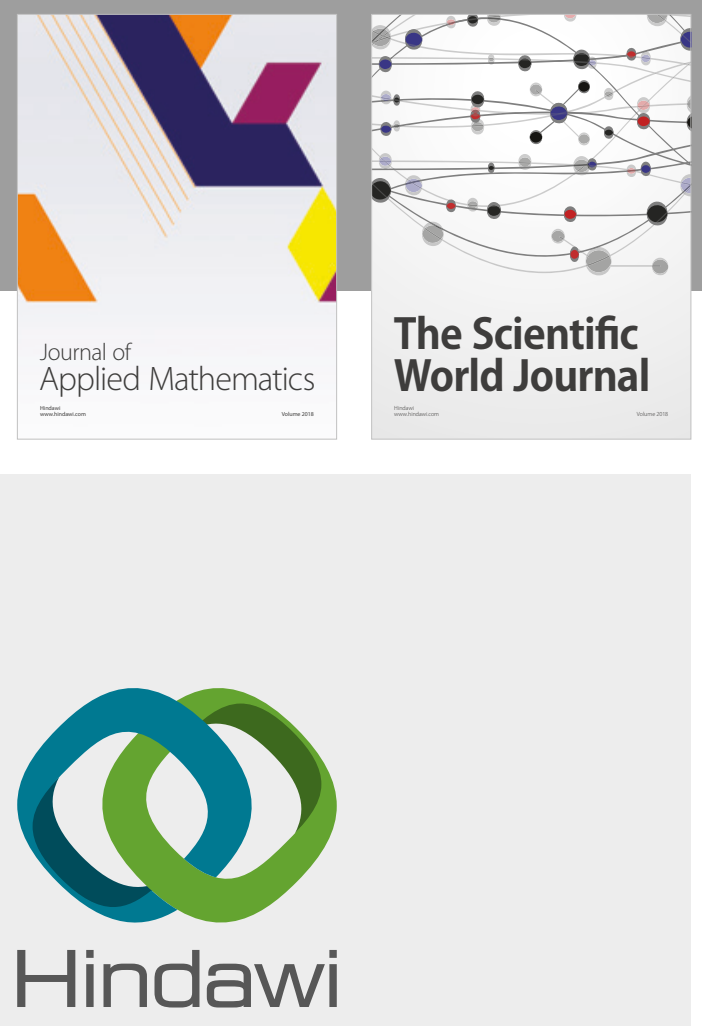

Submit your manuscripts at

www.hindawi.com

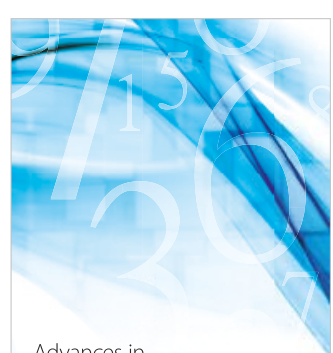

Advances in
Numerical Analysis
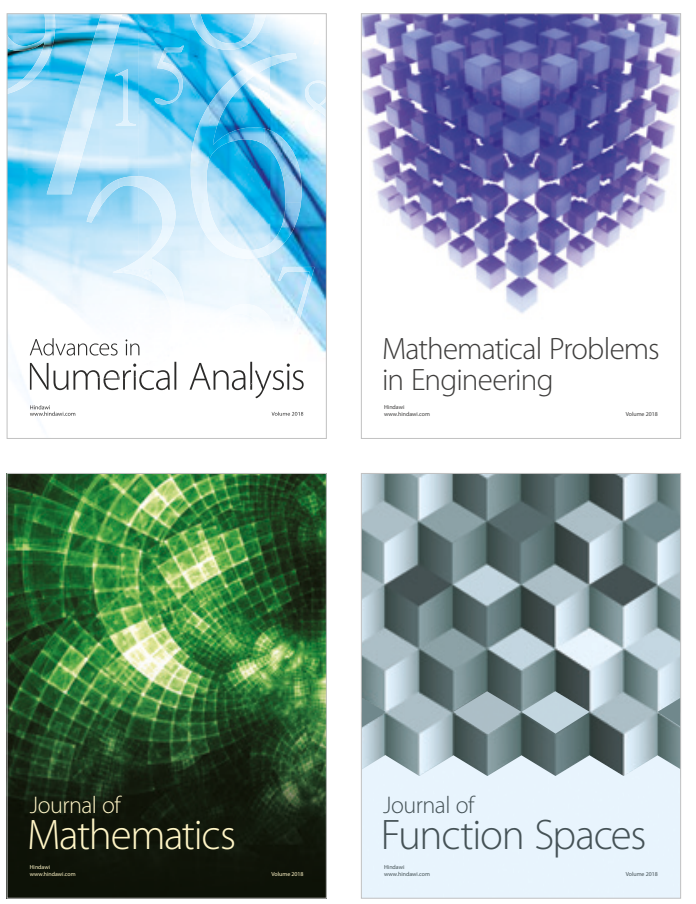

Mathematical Problems in Engineering

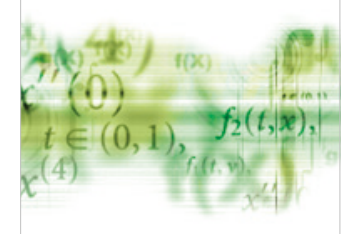

International Journal of

Differential Equations

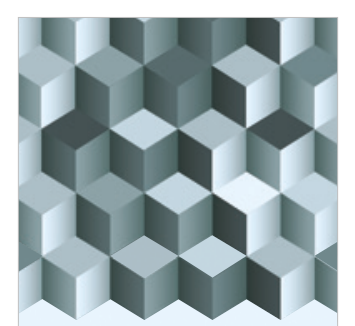

Journal of

Function Spaces
The Scientific

World Journal

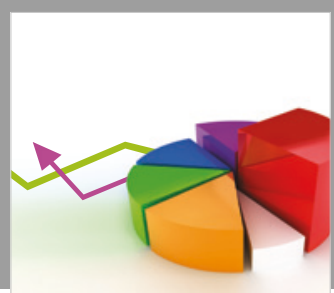

Journal of

Probability and Statistics
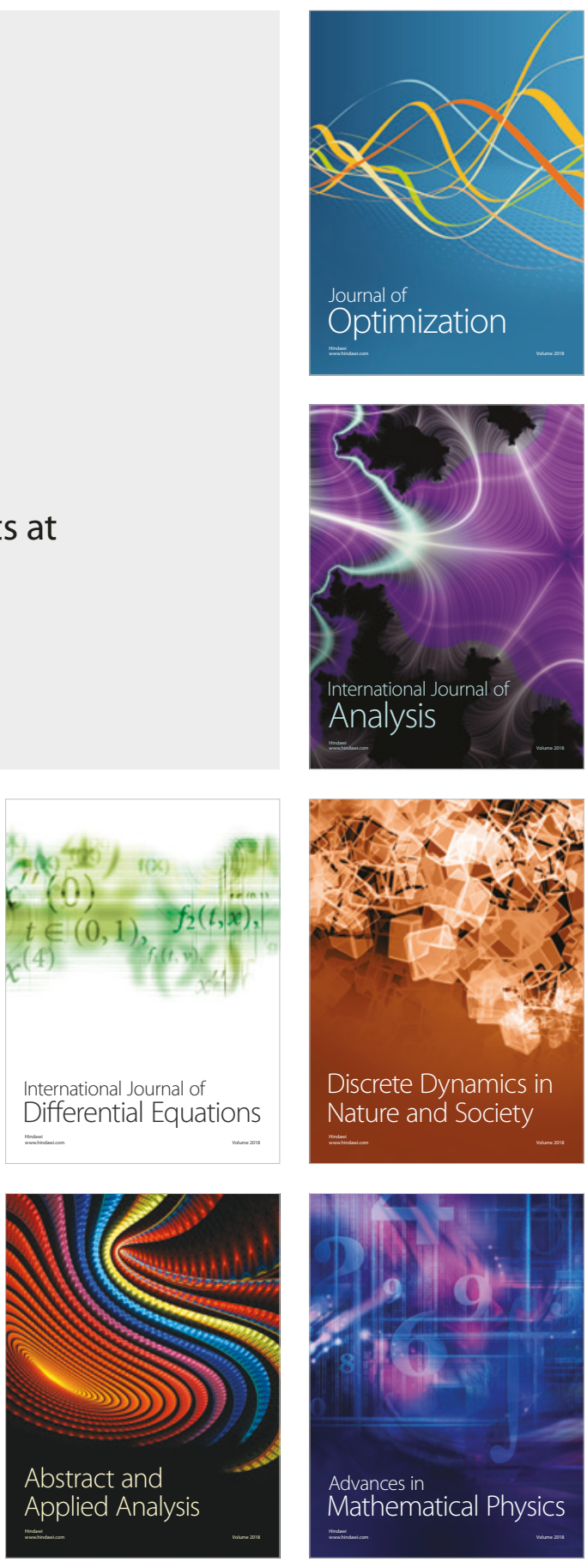